ARABIAN JOURNAL FOR SCIENCE AND ENGINEERING

ISSN: 2193-567X (print version); ISSN: 2191-4281 (electronic version)

Publisher: Springer; Impact factor $=1.092$

Accepted October $8^{\text {th }} 2018$

\title{
THERMAL SLIP IN OBLIQUE RADIATIVE NANO-POLYMER GEL TRANSPORT WITH TEMPERATURE-DEPENDENT VISCOSITY: SOLAR COLLECTOR NANOMATERIAL COATING MANUFACTURING SIMULATION
}

\author{
R. Mehmood ${ }^{1}$, Rabil Tabassum ${ }^{1 *}$, S. Kuharat ${ }^{2 \wedge}$, O. Anwar Bég ${ }^{2 *}$ and M. Babaie $^{3+}$ \\ ${ }^{1}$ Department of Mathematics, Faculty of Natural Sciences, HITEC University, Taxila Cantt, Pakistan. \\ ${ }^{2}$ Multi-Physical Engineering Sciences, Dept. Mechanical/Aeronautical Engineering, School of Computing, Science and \\ Engineering, Salford University, Manchester, M54WT, UK. \\ ${ }^{3}$ Energy Sciences, Petroleum and Gas Engineering Division, School of Computing, Science and Engineering, Salford \\ University, Manchester, M54WT, UK. \\ *Corresponding author: E-mail: rashid.mehmood@hitecuni.edu.pk,
}

\begin{abstract}
Nano-polymeric solar paints and sol-gels have emerged as a major new development in solar cell/collector coatings offering significant improvements in durability, anti-corrosion and thermal efficiency. They also exhibit substantial viscosity variation with temperature which can be exploited in solar collector designs. Modern manufacturing processes for such nano-rheological materials frequently employ stagnation flow dynamics under high temperature which invokes radiative heat transfer. Motivated by elaborating in further detail the nanoscale heat, mass and momentum characteristics, the present article presents a mathematical and computational study of the steady, two-dimensional, nonaligned thermo-fluid boundary layer transport of copper metal-doped water-based nano-polymeric sol gels under radiative heat flux. To simulate real nano-polymer boundary interface dynamics, thermal slip is analysed at the wall. A temperature-dependent viscosity is also considered. The conservation equations for mass, normal and tangential momentum and energy are normalized via appropriate transformations to generate a multi-degree, ordinary differential, non-linear, coupled boundary value problem. Numerical solutions are obtained via the stable, efficient Runge-Kutta-Fehlberg scheme with shooting quadrature in MATLAB symbolic software. Validation of solutions is achieved with a Variational Iterative Method (VIM) utilizing Langrangian multipliers. The impact of key emerging dimensionless parameters i.e. obliqueness parameter, radiation-conduction Rosseland number $(R d)$, thermal slip parameter $(\alpha)$, viscosity parameter $(m)$, nanoparticles volume fraction $(\phi)$ on nondimensional normal and tangential velocity components, temperature, wall shear stress, local heat flux and streamline distributions is visualized graphically. Shear stress and temperature are boosted with increasing radiative effect whereas local heat flux is reduced. Increasing wall thermal slip parameter depletes temperatures.
\end{abstract}

Keywords: non-orthogonal stagnation-point flow; thermal slip; variable viscosity; thermal radiation flux model; solar nano-polymer coating manufacture; copper volume fraction.

\section{INTRODUCTION}

In recent years nano-technology has had a profound impact on many technologies including medicine, aircraft components, rocket propulsion and also renewable energy systems [1]. In the latter area solar energy remains the best prospect for sustainable, clean, inexpensive power 
systems. Although significant progress has been made in refining the working fluids in solar collectors via nano-particle doping, only recently have engineers focused on revolutionizing the coating materials for solar cells and photovoltaics. Among the most promising of these developments is organic solar paint. This environmentally-friendly technology takes the form of coatings or flexible polymeric sheets that are precision-designed to contain a nano-particle fluid that is essentially water-based paint. The presence of the nano-particles has been confirmed to enhance durability, anti-corrosion and anti-abrasion characteristics of solar coatings which may be regarded as smart thermochromic materials [2]. With organic solar coatings, the solar collector (usually glass_-based housings) accumulate significantly less dry dust than untreated solar glass. Significant less dust on solar panels leads to less maintenance, lower cost per unit power generated over long periods of operation and higher overall energy production. Coating solar panels with these so-called sol gels and paints leads to a more consistent and predictable power output and sustained efficiency. These coating protects the glass from erosion, and from stubborn staining from salt and mineral deposits and furthermore have confirmed properties which can permit efficient performance in harsh environmental conditions from sub-zero to extreme hot environments. Solar gel coated systems constitute third generation solar designs (organic polymer-based nano-coatings) which are superceding the earlier first generation (silicon-based) and second generation (thin film) solar cells. Second Generation solar coatings have the disadvantage of susceptibility to contamination and also require very expensive and rare metals (Selenium, Cadmium, Tellerium etc).

Nano-polymeric solar gel coatings have several significant further advantages including solid active layers, anti-reflection properties, light weight and high flexibility, high transparency (active layer is approximately $100 \mathrm{~nm}$ ), can be 3-D printed and easily produced in large area and with low cost fabrication. Many excellent studies have been communicated on these novel solar gel coatings in recent years including Joly et al. [3] on copper, cobalt, magnesium and silicon hybrid doped water nan-solar gels. World-leading research has also been conducted at the California NanoSystems Institute of UCLA in the USA by Chen et al. [4] on the most advanced designs in transparent organic nano-polymer solar coatings. Important manufacturing studies have been reported also with regard to the high-quality synthesis of nano-solar gel coatings. Relevant studies in this regard include Joly et al. [5] on sol-gel dip stagnation flow coating, Schueller et al. [6] on multi-wafer sol-gel deposition fabrication and Joly et al. [7] on nano-gel coating production with both orthogonal and non-orthogonal stagnation fluid processes. 
The core science on which solar nano-gels are founded is that of nanofluids. Introduced by Argonne energy laboratory pioneers, Choi and Eastman [8] in the 1990s, a nanofluid is a suspension of nano-sized particles (usually metallic) introduced into a base fluid (water, ethylene glycol, air, lubricant etc) which boosts the effective thermal conductivity of the composite medium and even at relatively low volume fractions, the heat transfer enhancement is considerable. Many analytical studies of nanofluid dynamics have been communicated in the last decade or so. These have included external boundary layer flows, internal developing flows, swirling flows, squeezing flows, wedge (Falkner-Skan flows), peristaltic pumping, magnetohydrodynamics and helical flows. Many of these works have been reviewed recently by Bég [9]. The vast majority of these studies deploy either the Buongiorno two-component model (which invokes a species diffusion equation for nano-particles) or the simpler TiwariDas model (which allows volume fraction and different nanofluid properties to be simulated). A particular category of flows of special relevance to nanomaterial coating dynamics processes is stagnation-point flow. Such flows may involve either viscous (or inviscid) fluids impinging on solid surfaces and manifest in a vanishing of the local velocity and an associated peak in stagnation pressure. They are fundamental to materials processing operations but also arise in other areas of technology including aircraft wing aerodynamics, nuclear reactor ducts, polymer extrusion, vapour deposition in chemical engineering, analytical microfluidic chemistry (production and analysis of emulsions, single-cell systems, substrate patterning, and chipintegrated devices) [10] etc. Stagnation flows may be either orthogonal or non-orthogonal which refer respectively to the case where the impinging fluid is perpendicular or inclined (i.e. oblique) to the solid surface. Bachok et al. [11] investigated the time-dependent transverse nanofluid flow at a stagnation-point. They noted double solutions for values of unsteadiness constant less than zero. Uddin et al. [12] simulated stagnation-point bioconvection nanofluid flow with anisotropic hydrodynamic and thermal slip effects. Hamid et al. [13] considered nanofluid stagnation-point flow from a non-aligned pervious extending/shrinking sheet, noting that symmetry of the problem is disturbed by the transverse flow and that wall suction reduces the non-alignment of the flow.

In high-temperature materials processing, of which sol gel synthesis is an example, thermal radiation is significant. Generally, two modelling approaches are employed for simulating radiative heat transfer effects, namely linear and nonlinear models. Nonlinear radiation is valid for both high and low temperature differences whereas linear radiation is valid only for low temperature difference. Thermal radiation is critical in modern nano-polymer processing industry to control heat transfer processes which has a dramatic influence on the constitution 
of materials including nano-gels [14] and organic solar collector coatings [15]. Viskanta [16] has elaborated on the many complex phenomena intrinsic to radiative materials processing operations including the radiative properties of the material or nano-material (opaqueness, semi-transparency, absorption, reflection, transmissivity etc), spectral and directional radiation characteristics, radiative heat source (flames, lasers etc). The most general approach for simulating thermal radiative heat transfer is the integro-differential equation which is extremely difficult to solve in real applications [17]. Many simplifications of this general approach have been developed which broadly fall into two categories. The first, i.e. deterministic approach includes Hottel's Zone Method, Chandrasekhar's Discrete-Ordinate (Sn) Approximation and differential or algebraic flux models. A more rigorous approach is the statistical methodology which includes the Monte Carlo Ray-Trace Method (MCRT). In multi-physical fluid dynamics simulations, since many phenomena occur in conjunction with radiative heat transfer (e.g. convection, conduction, viscous flow etc), the most pragmatic approach has been to deploy algebraic flux models which include the Cogley-Vincenti-Giles non-gray flux model, MilneEddington flux model, Schuster-Schwartzchild two-flux model, Traugott P1 differential flux model and the Rosseland diffusion flux model [18]. The last of these models remains the most popular and although relatively simple, has been shown to valid for both gas and viscous liquid flows with high optical thickness. Rosselands's diffusion model leads to the incorporation of an extra derivative in the heat conservation equation and captures the physics quite well. It is based on the premise that optical thickness i.e. depth (a quantification of how opaque a medium is to radiation passing through it) is significantly larger than unity, for which the exponential integral in the radiative source decays speedily over a short geometrical distance) and furthermore that the fluid is a gray, absorbing-emitting but non-scattering media. Rosseland's flux model assumes an analogous form to the Fourier heat conduction equation and also the Fickian mass diffusion equation, making it more amenable for analysis. Numerous investigations have deployed this radiative flux model in multi-mode nanofluid mechanics. Ferdows et al. [19] analysed unsteady magnetized nanofluid extensional flow with wall suction and Rosseland radiative flux effects using an explicit finite difference procedure. Bég et al. [20] used network electro-thermal and spectral methods to study the thermos-solutal hydromagnetic time-dependent flow with Rosseland radiative flux and Soret/Dufour effects. Thumma et al. [21] used a Galerkin variational method to derive computational solutions for magnetized nanfoluid oscillatory oblique flow with radiative flux and thermo-physical effects. The rheological nature of nanofluids (e.g. sol gels) has also been established firmly by many key studies. Perse et al. [22] conducted detailed experiments on thickness sensitive spectrally 
selective (TSSS) coatings observing that they exhibit liquid-like viscoelastic behavior and are adequate mobility for proper leveling, uniform distribution of pigment particles inside dry coating and in addition achieve excellent solar absorptivity. Perse et al. [23] further conducted detailed tests on TISS sol-gel coatings, showing that rheology has a major influence on the spectral selectivity of TISS paints consisting of metallic nano-particles of Alumnium and small inorganic oxide pigment $(<500 \mathrm{~nm})$ embedded in a resin. Thermal radiation effects on rheological sol gel paints were considered in laboratory studies by Wijewardane and Goswami [24] and Atkinson et al. [25]. Many researchers have also employed a diverse spectrum of nonNewtonian models to analyse nanofluid flows. Uddin et al. [26] used MAPLE quadrature to simulate the power-law nanofluid transport in a porous matrix with Buongiorno's model. Bhatti and Rashidi [27] investigated thermo-diffusion and thermal radiation effects in rheological nanofluid flow from a stretching sheet using a successive linearization technique (SLM) and Chebyshev spectral collocation scheme (CSC). Hayat et al. [28] analyzed the Powell-Eyring nanoliquid flowing upon an elongating cylinder with magnetic field and thermal radiation. At the surface variation in concentration and temperature was taken into account. Huda et al. [29] employed the Reynolds variable-viscosity rheological model to compute the peristaltic flow and heat transfer of nano-polymers in biomimetic micro-pumps. Rana et al. [30] utilized the Reiner-Rivlin second grade fluid model and a finite element method to simulate dissipative rheo-nanofluid transport from an extruding sheet. Prasad et al. [31] employed an implicit finite difference method and Eringen's micropolar rheological model to study enrobing nanorheodynamics of a cylinder.

The above studies generally neglected slip phenomena at the nano-material/solid interface. Slip effects are known to arise in industrial polymeric systems including nano-gels and nanopolymers [32] and constitute non-adherence of fluids to boundaries at the molecular level. Even for the isotropic slip case the momentum and thermal characteristics may be dramatically modified at the wall. Various approaches have been developed to analyse both hydrodynamic (momentum) slip and this area has been extensively studied. However thermal slip which involves the inclusion of a slip parameter in the modified boundary conditions or the use of a Knudsen number has been less investigated in nano-polymer interfacial dynamics. Pal et al. [33] considered the dynamics of three different types of nanoliquids over an expanding sheet with thermal radiation and thermal slip conditions at the boundary, noting that temperature is depressed with a rise in thermal slip parameter whereas increasing hydrodynamic slip elevates temperatures. Latiff et al. [34] analysed the micropolar nanofluid 
time-dependent flow from a contracting/expanding sheet with gyrotactic micro-organism dynamics. Uddin et al. [35] studied numerically the bioconvection nanofluid boundary layer flow from a corrugated boundary with multiple slip effects. Turkyilmazoglu [36] presented asymptotic solutions for magnetic nanofluid flow thermosolutal convection boundary layers along extending/shrinking surfaces with thermal slip effects. Nagendra et al. [37] employed a Casson model to investigate magnetic viscoplastic nano-polymer enrobing flow on a vertical conical body with velocity and thermal slip effects. Ibrahim et al. [38] analyzed the radiativeconvective heat and mass transfer from a Newtonian nanofluid in Sakiadis flow with thermal and concentration slip effects. They showed that local Nusselt number is depleted with greater thermal slip effect whereas local Sherwood number is enhanced with increasing solutal slip. Hakeem et al. [39] computed the influence of second order velocity slip effect on magnetoconvective radiative, incompressible nanofluid extending/contracting sheet flow impact of magnetic field. At the boundary slip condition of second order is assumed. Dhana et al. [40] discussed the mixed convective flow of a nanoliquid with thermal slip condition over an inclined cylinder, highlighting that wall heat transfer rate is suppressed with a rise in thermal slip parameter whereas the opposite effect is induced in wall mass transfer rate. Prabhakar et al. [41] studied reactive magnetic viscoplastic nanofluid flow from an exponentiallyelongating sheet subjected to oblique magnetic field and wall momentum and thermal slip. In the present article we investigate time-independent two-dimensional, non-aligned (oblique) slip flow of copper metal-doped water-based nano-polymeric sol gels under radiative heat flux. Reynolds temperature-dependent viscosity model is employed as are the Tiwari-Das nanofluid volume fraction model and Maxwell-Garnet model for the nanofluid thermal conductivity. Such a simulation, although greatly relevant to nano-sol gel manufacturing processes has not thusfar received any attention in the literature. The transformed ordinary differential conservation equations are solved with the Runge-Kutta-Fehlberg scheme and shooting quadrature in MATLAB symbolic software. Validation of solutions is conducted with a Variational Iterative Method (VIM). The response in non-dimensional normal and tangential velocity components, temperature, wall shear stress, local heat flux and streamline distributions to variation in obliqueness parameter, radiation-conduction Rosseland number, thermal slip parameter, Reynolds viscosity parameter, nanoparticles volume fraction is described and interpreted at length. It is envisaged that the current effort will provide a good compliment to experimental and more complex computational fluid dynamics simulations of solar collector gel coating synthesis [42]. 


\section{MATHEMATICAL MODEL}

Two-dimensional, oblique stagnation flow of viscous, incompressible variable viscosity sol gel nano-polymer under steady-state conditions is considered. The objective is to model the manufacturing dynamics of sol gel copper-based nano-polymer paints which are used in organic third generation solar collectors. Interfacial slip is analyzed via a thermal slip boundary condition. All other properties of the nano-polymer are taken as constant except viscosity which is temperature-dependent and simulated via Reynolds model. High-temperature invokes appreciable thermal radiative heat transfer. To facilitate numerical analysis of the radiative contribution, only unidirectional radiation flux, $q^{*}$, is considered and it is assumed that $\frac{\partial \widehat{\mathrm{q}}_{\mathrm{r}}{ }^{*}}{\partial \widehat{\mathrm{y}}^{*}}$ >> $\frac{\partial \widehat{\mathrm{q}}_{\mathrm{r}}{ }^{*}}{\partial \mathrm{x}}{ }^{*}$. This model assumes that the radiative intensity is the actual black-body intensity at the nano-polymer temperature. The principal basis for this approximation is to consider that the irradiation is diffused inside the medium and that optical properties inside the polymer are incorporated via the Rosseland conductivity, as elaborated by Cess [43]. The effect of radiation is manifested in the form of enhanced thermal diffusivity. The radiative heat flux component on the wall is expressed with the help of Stefan-Boltzmann law. The Rosseland approximation permits the simplification of the governing integro-differential equation for radiative energy balance into a Fourier-type diffusion equation analogous to that describing heat conduction, potential flow or electrostatic potential (Coulomb's law) which is valid for optically-thick media in which radiation only propagates a limited distance prior to experiencing scattering or absorption. The local intensity is generated by thermal radiation emanating from nearby locations in the vicinity of which the emission and scattering are comparable to the location under consideration. For zones where conditions are appreciably different, the radiation has been shown to be greatly attenuated prior to arriving at the location being analyzed. The energy transfer depends only on the conditions in the area near the position under consideration. Intrinsic to Rosseland's model is that the radiation intensity is uniform and isotropic in the nano-polymer, refractive index is invariant and medium optical thickness exceeds three. It is also pertinent to note that the Rosseland model is a special case of the more elaborate P1 differential approximation [44] which itself is obtained by a truncated expansion of the general radiative equation to fourth order terms. Although we restrict attention to isotropic nanopolymers, the Rosseland model does allow for anisotropic scattering using a phase function and this is the subject of a subsequent study [45]. Justification for employing Rosseland's 
model is strong as endorsed by excellent studies on radiative thermo-coating polymer dynamics where the model has been shown to yield sufficiently accurate results but with less computational expense and more easy incorporation into coupled nonlinear convectiveconductive-radiative transport [46, 47]. It has however been identified that since the Rosseland diffusion approximation is not valid at the interface (wall), it is recommended to deploy a temperature slip boundary condition, as in the present model [48] which is accommodated well by the incompressible laminar boundary layer theory (as considered in the present oblique stagnation flow regime). Two equal and opposite forces are applied along the $\hat{\mathrm{x}}^{*}$-axis in order to extend (stretch) the nano-polymer surface, with the origin fixed. The two-dimensional model for the sol gel nano-polymer stagnation flow problem is displayed in Fig. (1). Gravity force acts in the negative $\hat{\mathrm{x}}^{*}$-direction. The nano-polymer is assumed to be gray, and emits and absorbs but does not scatter radiation. Since stagnation flows are typically slow and viscousdominated, viscous dissipation may be neglected. The nano-polymer is assumed to be a dilute suspension containing equal sized copper nano-particles and ballistic collisions are ignored. The basic model equations for the steady-state problem in an $\left(\hat{\mathrm{x}}^{*}, \hat{\mathrm{y}}^{*}\right)$ coordinate system, are the conservation laws of mass, momentum, energy which may be formulated as follows:

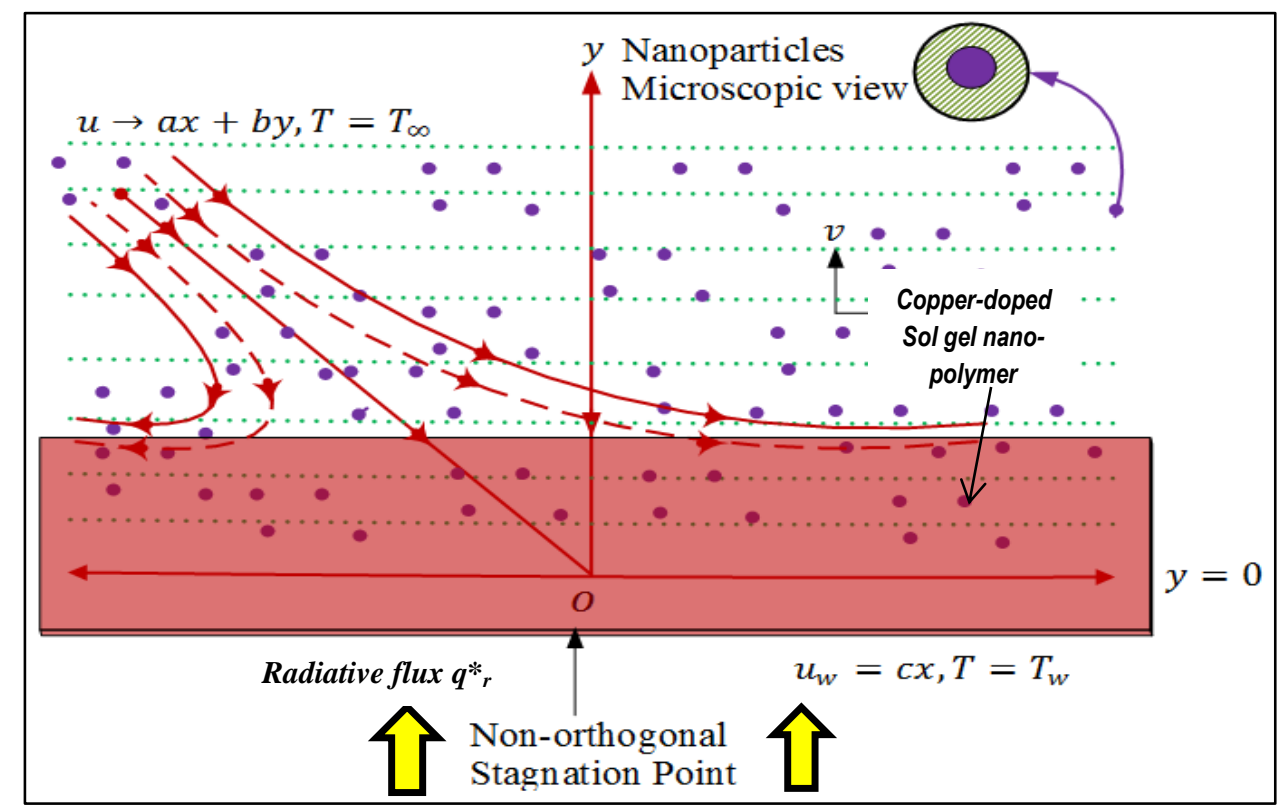

Fig. (1). Physical description of sol gel nano-polymer oblique stagnation flow processing.

$$
\frac{\partial \widehat{\mathrm{u}}^{*}}{\partial \widehat{\mathrm{x}}^{*}}+\frac{\partial \widehat{\mathrm{v}}^{*}}{\partial \widehat{\mathrm{y}}^{*}}=0
$$


$\widehat{\mathrm{u}}^{*} \frac{\partial \widehat{\mathrm{u}}^{*}}{\partial \widehat{\mathrm{x}}^{*}}+\widehat{\mathrm{v}}^{*} \frac{\partial \widehat{\mathrm{u}}^{*}}{\partial \widehat{\mathrm{y}}^{*}}+\frac{1}{\widehat{\hat{\rho}}_{\mathrm{nf}}} \frac{\partial \widehat{\mathrm{p}}^{*}}{\partial \widehat{\mathrm{x}}^{*}}=\frac{\mu_{\mathrm{nf}}\left(\widehat{\mathrm{T}}^{*}\right)}{\widehat{\rho}_{\mathrm{nf}}} \frac{\partial^{2} \widehat{\mathrm{u}}^{*}}{\partial \widehat{\mathrm{y}}^{*}}+\frac{1}{\widehat{\hat{\rho}}_{\mathrm{nf}}} \frac{\partial \widehat{\mathrm{u}}^{*}}{\partial \widehat{\mathrm{y}}^{*}} \frac{\partial \mu_{\mathrm{nf}}\left(\widehat{\mathrm{T}}^{*}\right)}{\partial \widehat{\mathrm{T}}^{*}} \frac{\partial \widehat{\mathrm{T}}^{*}}{\partial \widehat{\mathrm{y}}^{*}}$,

$\widehat{\mathrm{u}}^{*} \frac{\partial \widehat{\mathrm{v}}^{*}}{\partial \widehat{\mathrm{x}}^{*}}+\widehat{\mathrm{v}}^{*} \frac{\partial \widehat{\mathrm{v}}^{*}}{\partial \widehat{\mathrm{y}}^{*}}+\frac{1}{\widehat{\rho}_{\mathrm{nf}}} \frac{\partial \widehat{\mathrm{p}}^{*}}{\partial \widehat{\mathrm{y}}^{*}}=\frac{\mu_{\mathrm{nf}}\left(\widehat{\mathrm{T}}^{*}\right)}{\widehat{\rho}_{\mathrm{nf}}} \frac{\partial^{2} \widehat{\mathrm{v}}^{*}}{\partial \widehat{\mathrm{y}}^{*}}+\frac{1}{\widehat{\rho}_{\mathrm{nf}}} \frac{\partial \widehat{\mathrm{v}}^{*}}{\partial \widehat{\mathrm{y}}^{*}} \frac{\partial \mu_{\mathrm{nf}}\left(\widehat{\mathrm{T}}^{*}\right)}{\partial \widehat{\mathrm{T}}^{*}} \frac{\partial \widehat{T}^{*}}{\partial \widehat{\mathrm{y}}^{*}}$,

$\widehat{\mathrm{u}}^{*} \frac{\partial \widehat{\mathrm{T}}^{*}}{\partial \widehat{\mathrm{x}}^{*}}+\widehat{\mathrm{v}}^{*} \frac{\partial \widehat{\mathrm{T}}^{*}}{\partial \widehat{\mathrm{y}}^{*}}=\widehat{\alpha}_{\mathrm{nf}}^{*} \frac{\partial^{2} \widehat{\mathrm{T}}^{*}}{\partial \widehat{\mathrm{y}}^{*^{2}}}-\frac{1}{\left(\widehat{\rho} \mathrm{c}_{\widehat{\mathrm{p}}}\right)_{\mathrm{nf}}} \frac{\partial \widehat{\mathrm{q}}_{\mathrm{r}}{ }^{*}}{\partial \widehat{\mathrm{y}}^{*}}$,

Here $\mu_{\mathrm{nf}}\left(\widehat{\mathrm{T}}^{*}\right)=\frac{\mu_{0} \mathrm{e}^{-\mathrm{d}\left(\widehat{\mathrm{T}}^{*}-\widehat{\mathrm{T}}_{\infty}\right)}}{(1-\phi)^{2.5}}$ is the nano-particle modified viscosity function based on Reynolds model in which the viscosity varies exponentially with temperature and $d$ is Reynolds viscosity variation exponent, $\widehat{T}^{*}$ denotes the temperature, $\phi$ indicates the nanoparticles volume fraction, $\mu_{0}$ is the reference viscosity and $\widehat{T}_{\infty}$ signifies the ambient temperature of nanofluid. In eqns. (1)-(4), $\hat{\mathrm{u}}^{*}, \hat{\mathrm{v}}^{*}$ are $\hat{\mathrm{x}}^{*}, \hat{\mathrm{y}}^{*}$ components of velocity, $\hat{\mathrm{p}}^{*}$ represents the pressure, $\hat{\rho}_{\mathrm{nf}}$ denotes the nanofluid (sol gel nano-polymer) density and $\widehat{\alpha}_{\mathrm{nf}}^{*}=\frac{\widehat{\mathrm{k}}_{\mathrm{nf}}}{\left(\widehat{\rho}_{\widehat{\mathrm{p}}}\right)_{\mathrm{nf}}}$ signifies the effective thermal diffusivity of nanofluid. $\widehat{\mathrm{q}}_{\mathrm{r}}^{*}=-\left(\frac{16 \widehat{\delta}^{*} \widehat{\mathrm{T}}_{\infty}^{3}}{3 \mathrm{~K}^{*}}\right) \frac{\partial \widehat{\mathrm{T}}^{*}}{\partial \widehat{\mathrm{y}}^{*}}$ characterizes the radiative thermal linearized heat flux with $\hat{\delta}^{*}$ and $K^{*}$ denoting Stefan-Boltzmann constant and Rosseland mean absorption coefficient. Optical properties inside the nano-polymer are taken into account via the Rosseland conductivity which is defined by $k_{\text {Ross }}=\frac{16 \widehat{\delta}^{*} \widehat{T}_{\infty}^{3}}{3 K^{*}}$. It is important to note that $K^{*}$ i.e. Rosseland mean absorption coefficient is derived analytically as a function of temperature via integration over the frequency [46]. Furthermore, the nanofluid effective density and thermal capacitance are given by the following formulae [47]:

$\hat{\rho}_{\mathrm{nf}}=(1-\phi) \hat{\rho}_{\mathrm{f}}+\phi \hat{\rho}_{\mathrm{s}}$

$\left(\hat{\rho} c_{\widehat{p}}\right)_{n f}=(1-\phi)\left(\hat{\rho} c_{\widehat{p}}\right)_{f}+\phi\left(\hat{\rho} c_{\widehat{p}}\right)_{s^{\prime}}$

Here $\hat{\rho}_{\mathrm{s}}$ and $\hat{\rho}_{\mathrm{f}}$ indicate the densities of nanoparticles and base fluid respectively. Nanopolymer thermal conductivity, $\hat{\mathrm{k}}_{\mathrm{nf}}$ is approximated by employing the Maxwell-Garnet model $[47,48]$ defined as:

$\frac{\hat{\mathrm{k}}_{\mathrm{nf}}}{\hat{\mathrm{k}}_{\mathrm{f}}}=\frac{\hat{\mathrm{k}}_{\mathrm{s}}+2 \hat{\mathrm{k}}_{\mathrm{f}}-2 \phi\left(\hat{\mathrm{k}}_{\mathrm{f}}-\hat{\mathrm{k}}_{\mathrm{s}}\right)}{\hat{\mathrm{k}}_{\mathrm{s}}+2 \hat{\mathrm{k}}_{\mathrm{f}}+\phi\left(\hat{\mathrm{k}}_{\mathrm{f}}-\hat{\mathrm{k}}_{\mathrm{s}}\right)}$, 
Here thermal conductivities of the carrier (base) fluid i.e. water and the copper nanoparticles are denoted by $\hat{\mathrm{k}}_{\mathrm{f}}$ and $\hat{\mathrm{k}}_{\mathrm{s}}$. Some relevant properties of copper and water are shown as below:

\begin{tabular}{|l|c|c|c|}
\hline \multicolumn{1}{|c|}{$\begin{array}{c}\text { Thermo-physical } \\
\text { properties }\end{array}$} & $\hat{\boldsymbol{\rho}}\left(\frac{\mathbf{k g}}{\mathbf{m}^{\mathbf{3}}}\right)$ & $\mathbf{c}_{\widehat{\mathbf{p}}}\left(\frac{\mathbf{J}}{\mathbf{k g} \cdot \mathbf{K}}\right)$ & $\hat{\mathbf{k}}\left(\frac{\mathbf{W}}{\mathbf{m} \cdot \mathbf{K}}\right)$ \\
\hline $\mathbf{H}_{\mathbf{2}} \mathbf{O}$ & 997.1 & 4179 & 0.613 \\
\hline $\mathbf{C u}$ & 8933 & 385 & 400 \\
\hline
\end{tabular}

Table. 1: Thermo-physical characteristics of copper-water sol gel nano-polymer

The following boundary conditions are enforced at the wall (nano-polymer-solid interface) and in the free stream:

$$
\begin{gathered}
\hat{\mathrm{u}}^{*}=c \hat{\mathrm{x}}^{*}, \hat{\mathrm{v}}^{*}=0, \widehat{\mathrm{T}}^{*}=\widehat{\mathrm{T}}_{\mathrm{w}}+\widehat{\mathrm{D}} \frac{\partial \widehat{\mathrm{T}}^{*}}{\partial \widehat{\mathrm{y}}^{*}} \text { at } \hat{\mathrm{y}}^{*}=0, \\
\hat{\mathrm{u}}^{*}=\mathrm{a} \hat{\mathrm{x}}^{*}+\mathrm{b} \hat{\mathrm{y}}^{*}, \widehat{\mathrm{T}}^{*}=\widehat{\mathrm{T}}_{\infty} \quad \text { as } \hat{\mathrm{y}}^{*} \rightarrow \infty .
\end{gathered}
$$

Here $\widehat{\mathrm{D}}$ is thermal slip factor, $a, b$ and $c$ are constants with inverse time dimensions and $\widehat{\mathrm{T}}_{\mathrm{w}}$ signifies the wall temperature. To normalize the model, it is pertinent to invoke the following similarity transformations:

$\hat{x}=\hat{x}^{*} \sqrt{\frac{c}{\hat{v}_{f}}}, \hat{y}=\hat{y}^{*} \sqrt{\frac{c}{\hat{v}_{f}}}, \hat{u}=\hat{u}^{*} \frac{1}{\sqrt{\hat{v}_{f} c}}, \hat{v}=\hat{v}^{*} \frac{1}{\sqrt{\hat{v}_{\mathrm{f}}}}, \hat{p}=\frac{\widehat{p}^{*}}{(\widehat{\rho} \hat{v})_{\mathrm{f}} \mathrm{c}}, \widehat{T}=\frac{\widehat{T}^{*}-\widehat{T}_{\infty}}{\widehat{T}_{\mathrm{w}}-\widehat{T}_{\infty}}$,

Here the effective kinematic viscosity of the base fluid (water) is denoted by $\hat{v}_{\mathrm{f}}$. Implementing the transformations given in Eqn. (10), into the nano-polymer boundary layer Eqns. (1) (4), with associated boundary conditions (8) - (9) yields the following dimensionless partial differential boundary value problem:

$$
\frac{\partial \widehat{u}}{\partial \hat{x}}+\frac{\partial \widehat{v}}{\partial \widehat{y}}=0,
$$

$\hat{\mathrm{u}} \frac{\partial \widehat{u}}{\partial \widehat{\mathrm{x}}}+\widehat{v} \frac{\partial \widehat{u}}{\partial \widehat{y}}+\frac{\widehat{\rho}_{\mathrm{f}}}{\widehat{\rho}_{\mathrm{nf}}} \frac{\partial \widehat{\mathrm{p}}}{\partial \widehat{\mathrm{x}}}=\frac{\widehat{\rho}_{\mathrm{f}}}{\widehat{\rho}_{\mathrm{nf}}}\left(\frac{\mathrm{e}^{-\mathrm{m} \widehat{\mathrm{T}}}}{(1-\phi)^{2.5}}\left(\frac{\partial^{2} \widehat{\mathrm{u}}}{\partial \widehat{y}^{2}}-\mathrm{m} \frac{\partial \widehat{u}}{\partial \widehat{y}} \frac{\partial \widehat{\mathrm{T}}}{\partial \widehat{y}}\right)\right)$,

$$
\widehat{\mathrm{u}} \frac{\partial \widehat{\mathrm{v}}}{\partial \hat{\mathrm{x}}}+\widehat{\mathrm{v}} \frac{\partial \widehat{\mathrm{v}}}{\partial \widehat{\mathrm{y}}}+\frac{\widehat{\rho}_{\mathrm{f}}}{\widehat{\rho}_{\mathrm{nf}}} \frac{\partial \widehat{\mathrm{p}}}{\partial \widehat{\mathrm{y}}}=\frac{\widehat{\rho}_{\mathrm{f}}}{\widehat{\rho}_{\mathrm{nf}}}\left(\frac{\mathrm{e}^{-\mathrm{m} \widehat{T}}}{(1-\phi)^{2.5}}\left(\frac{\partial^{2} \widehat{\mathrm{v}}}{\partial \widehat{\mathrm{y}}^{2}}-\mathrm{m} \frac{\partial \widehat{\mathrm{v}}}{\partial \widehat{\mathrm{y}}} \frac{\partial \widehat{\mathrm{T}}}{\partial \widehat{\mathrm{y}}}\right)\right),
$$


$\hat{\mathrm{u}} \frac{\partial \widehat{\mathrm{T}}}{\partial \widehat{\mathrm{x}}}+\hat{\mathrm{v}} \frac{\partial \widehat{\mathrm{T}}}{\partial \widehat{y}}=\left(\frac{\widehat{\alpha}_{\mathrm{nf}}}{\widehat{v}_{\mathrm{f}}}+\frac{16 \widehat{\delta}^{*} \mathrm{~T}_{\infty}^{3}}{3 \widehat{v}_{\mathrm{f}}\left(\widehat{\rho}_{\widehat{\mathrm{p}}}\right)_{\mathrm{nf}} \mathrm{K}^{*}}\right) \frac{\partial^{2} \widehat{T}}{\partial \widehat{\mathrm{y}}^{2}}$

$\hat{\mathrm{u}}=\hat{\mathrm{x}}, \hat{\mathrm{v}}=0, \widehat{\mathrm{T}}=1+\alpha \frac{\partial \widehat{\mathrm{T}}}{\partial \widehat{\mathrm{y}}}$ at $\hat{\mathrm{y}}=0$,

$\hat{\mathrm{u}}=\frac{\mathrm{a}}{\mathrm{c}} \hat{\mathrm{x}}+\frac{\mathrm{b}}{\mathrm{c}} \hat{\mathrm{y}}, \widehat{\mathrm{T}}=0$ at $\hat{\mathrm{y}} \rightarrow \infty$.

The new parameter $m=\mathrm{d}\left(\widehat{\mathrm{T}}_{\mathrm{w}}-\widehat{\mathrm{T}}_{\infty}\right)$ represents the Reynolds viscosity parameter (for nanopolymer rheology) and $\alpha=\widehat{\mathrm{D}} \sqrt{\frac{\mathrm{c}}{\hat{v}_{\mathrm{f}}}}$ is the thermal slip parameter. It is further of benefit to define a dimensional stream function as follows [49]:

$\widehat{\mathrm{u}}=\frac{\partial \psi}{\partial \widehat{\mathrm{y}}}, \hat{\mathrm{v}}=-\frac{\partial \psi}{\partial \widehat{\mathrm{x}}}$.

Using the above relation in Eqns. (11) - (16) and eliminating the pressure term $\hat{p}$ by virtue of the fact that $\hat{p}_{\hat{x} \hat{y}}=\hat{p}_{\hat{y} \hat{x}}$, the following equations emerge:

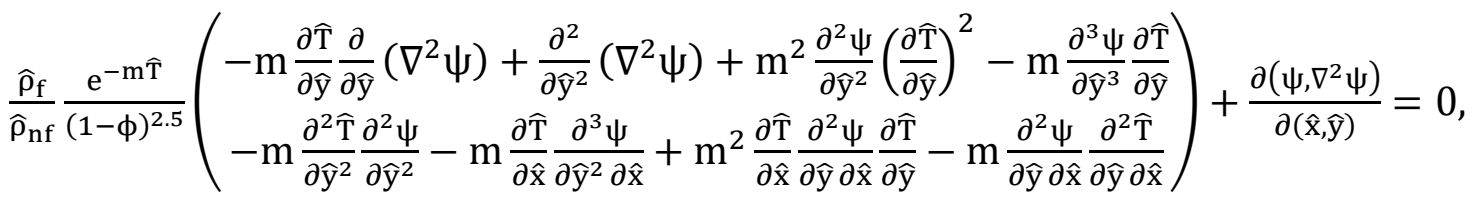

$\frac{\partial \psi}{\partial \widehat{y}} \frac{\partial \widehat{T}}{\partial \hat{x}}-\frac{\partial \Psi}{\partial \hat{x}} \frac{\partial \widehat{T}}{\partial \widehat{y}}=\left(\frac{\widehat{\alpha}_{n f}}{\widehat{v}_{f}}+\frac{16 \widehat{\delta}^{*} T_{\infty}^{3}}{3 \widehat{v}_{f}\left(\widehat{\rho}_{\widehat{p}}\right)_{n f} K^{*}}\right) \frac{\partial^{2} \widehat{T}}{\partial \widehat{y}^{2}}$

$\psi=0, \frac{\partial \psi}{\partial \widehat{y}}=\hat{x}, \widehat{T}=1+\alpha \frac{\partial \widehat{T}}{\partial \widehat{y}}$ at $\hat{y}=0$,

$\psi=\frac{\mathrm{a}}{\mathrm{c}} \hat{\mathrm{x}} \hat{\mathrm{y}}+\frac{1}{2} \gamma \hat{\mathrm{y}}^{2}, \widehat{\mathrm{T}}=0$ at $\hat{\mathrm{y}} \rightarrow \infty$.

Here $\gamma=\frac{\mathrm{b}}{\mathrm{c}}$ signifies the obliqueness parameter i.e. secondary (tangential) stretching ratio. Stream function is re-defined [49] as:

$\psi(\hat{x}, \hat{y})=\hat{x} F(\hat{y})+G(\hat{y}), \widehat{T}(\hat{x}, \hat{y})=\theta(\hat{y})$, 
$\mathrm{F}(\hat{\mathrm{y}})$ and $\mathrm{G}(\hat{\mathrm{y}})$ are normal and tangential (oblique) velocity components. Introducing Eqn. (22) in the Eqns. (18) - (21), with subsequent integration, generates the following nondimensional, eighth-order system of coupled, nonlinear, multi-degree ordinary differential equations system:

\section{Normal momentum}

$\frac{\hat{\rho}_{\mathrm{f}}}{\hat{\rho}_{\mathrm{nf}}} \frac{\mathrm{e}^{-\mathrm{m} \theta}}{(1-\phi)^{2.5}}\left(\mathrm{~F}^{\prime \prime \prime}-\mathrm{m} \theta^{\prime} \mathrm{F}^{\prime \prime}\right)+\mathrm{FF}^{\prime \prime}-\left(\mathrm{F}^{\prime}\right)^{2}+\mathrm{C}_{1}=0$,

\section{Tangential momentum}

$\frac{\widehat{\rho}_{\mathrm{f}}}{\widehat{\rho}_{\mathrm{nf}}} \frac{\mathrm{e}^{-\mathrm{m} \theta}}{(1-\phi)^{2.5}}\left(\mathrm{G}^{\prime \prime \prime}-\mathrm{m} \theta^{\prime} \mathrm{G}^{\prime \prime}\right)+\mathrm{FG}^{\prime \prime}-\mathrm{F}^{\prime} \mathrm{G}^{\prime}+\mathrm{C}_{2}=0$,

\section{Energy (heat)}

$\left(\frac{\widehat{k}_{\mathrm{nf}}}{\widehat{\mathrm{k}}_{\mathrm{f}}}+\frac{4}{3} \mathrm{Rd}\right) \theta^{\prime \prime}+\operatorname{Pr}\left(1-\phi+\frac{\left(\hat{\rho} c_{\widehat{\mathrm{p}}}\right)_{\mathrm{s}}}{\left(\hat{\mathrm{\rho}} \mathrm{c}_{\widehat{\mathrm{p}}}\right)_{\mathrm{f}}} \phi\right) \mathrm{F} \theta^{\prime}=0$

Here $C_{1}$ and $C_{2}$ are integration constants and derivatives with respect to $\hat{y}$ are designated by primes. $R d=\frac{4 \widehat{\delta}^{*} T_{\infty}^{3}}{\widehat{\mathrm{k}}_{\mathrm{f}} \mathrm{K}^{*}}$ signifies the radiation-conduction parameter (also known as Stark number or Rosseland-Boltzmann number). The boundary conditions are transformed as:

$\left.\begin{array}{c}F(0)=0, F^{\prime}(0)=1, G^{\prime}(0)=0, \\ F^{\prime}(\infty)=\frac{a}{c}, G^{\prime \prime}(\infty)=\gamma, \theta(0)=1+\alpha \theta^{\prime}(0), \theta(\infty)=0\end{array}\right\}$.

In the limit, $\hat{y} \rightarrow \infty$, in Eq. (23) and with the boundary condition $\mathrm{F}^{\prime}(\infty)=\frac{\mathrm{a}}{\mathrm{c}}$ we get $C_{1}=$ $\left(\frac{a}{c}\right)^{2} \cdot F(\hat{y})=\left(\frac{a}{c}\right) \hat{y}+A$ is obtained by analysing the boundary layer Eq. (23) when $\hat{y} \rightarrow \infty$, where the constant $A$ designates boundary layer displacement. Similarly using $G^{\prime \prime}(\infty)=\gamma$ in Eq. (24) after applying the limit $\hat{y} \rightarrow \infty$, we obtain $C_{2}=-A \gamma$. Hence Eqns. (23) and (24) become:

$$
\begin{aligned}
& \frac{\mathrm{e}^{-\mathrm{m} \theta}}{(1-\phi)^{2.5}}\left(\mathrm{~F}^{\prime \prime \prime}-\mathrm{m} \theta^{\prime} \mathrm{F}^{\prime \prime}\right)+\frac{\hat{\rho}_{\mathrm{nf}}}{\hat{\rho}_{\mathrm{f}}}\left(\mathrm{FF}^{\prime \prime}-\left(\mathrm{F}^{\prime}\right)^{2}+\left(\frac{\mathrm{a}}{\mathrm{c}}\right)^{2}\right)=0, \\
& \frac{\mathrm{e}^{-\mathrm{m} \theta}}{(1-\phi)^{2.5}}\left(\mathrm{G}^{\prime \prime \prime}-\mathrm{m} \theta^{\prime} \mathrm{G}^{\prime \prime}\right)+\frac{\hat{\rho}_{\mathrm{nf}}}{\hat{\rho}_{\mathrm{f}}}\left(F G^{\prime \prime}-\mathrm{F}^{\prime} \mathrm{G}^{\prime}-\mathrm{A} \gamma\right)=0,
\end{aligned}
$$


Proceeding with the analysis, introducing the relation:

$G^{\prime}(\hat{y})=\gamma H(\hat{y})$

Using Eq. (29) in (28) leads to:

$\frac{\mathrm{e}^{-\mathrm{m} \theta}}{(1-\phi)^{2.5}}\left(\mathrm{H}^{\prime \prime}-\mathrm{m} \theta^{\prime} \mathrm{H}^{\prime}\right)+\frac{\widehat{\rho}_{\mathrm{nf}}}{\widehat{\rho}_{\mathrm{f}}}\left(\mathrm{FH} \mathrm{H}^{\prime}-\mathrm{F}^{\prime} \mathrm{H}-\mathrm{A}\right)=0$,

The associated tangential velocity boundary conditions emerge as:

$\mathrm{H}(0)=0, \mathrm{H}^{\prime}(\infty)=1$.

Therefore the final ordinary differential boundary value to be solved reduces in summary to:

$\frac{\mathrm{e}^{-\mathrm{m} \theta}}{(1-\phi)^{2.5}}\left(\mathrm{~F}^{\prime \prime \prime}-\mathrm{m} \theta^{\prime} \mathrm{F}^{\prime \prime}\right)+\frac{\hat{\rho}_{\mathrm{nf}}}{\hat{\rho}_{\mathrm{f}}}\left(\mathrm{FF}^{\prime \prime}-\left(\mathrm{F}^{\prime}\right)^{2}+\left(\frac{\mathrm{a}}{\mathrm{c}}\right)^{2}\right)=0$,

$\frac{\mathrm{e}^{-\mathrm{m} \theta}}{(1-\phi)^{2.5}}\left(\mathrm{H}^{\prime \prime}-\mathrm{m} \theta^{\prime} \mathrm{H}^{\prime}\right)+\frac{\widehat{\rho}_{\mathrm{nf}}}{\widehat{\rho}_{\mathrm{f}}}\left(\mathrm{FH} \mathrm{H}^{\prime}-\mathrm{F}^{\prime} \mathrm{H}-\mathrm{A}\right)=0$,

$\left(\frac{\widehat{\mathrm{k}}_{\mathrm{nf}}}{\widehat{\mathrm{k}}_{\mathrm{f}}}+\frac{4}{3} \mathrm{Rd}\right) \theta^{\prime \prime}+\operatorname{Pr}\left(1-\phi+\frac{\left(\widehat{\rho} \mathrm{c}_{\widehat{\mathrm{p}}}\right)_{\mathrm{s}}}{\left(\widehat{\rho}_{\hat{\mathrm{p}}}\right)_{\mathrm{f}}} \phi\right) \mathrm{F} \theta^{\prime}=0$.

$\left.\begin{array}{c}\mathrm{F}(0)=0, \mathrm{~F}^{\prime}(0)=1, \mathrm{H}(0)=0, \\ \mathrm{~F}^{\prime}(\infty)=\frac{\mathrm{a}}{\mathrm{c}}, \mathrm{H}^{\prime}(\infty)=1, \theta(0)=1+\alpha \theta^{\prime}(0), \theta(\infty)=0\end{array}\right\}$.

In the above system, $a / c$ is the primary (normal) sheet stretching ratio, A i.e. the boundary layer displacement is computed as explained from the condition, $F(\hat{y})=\left(\frac{a}{c}\right) \hat{y}+A$ when $\hat{y} \rightarrow$ $\infty$, and is internally computed in Matlab numerical quadrature during the execution of the program (since this so-called constant actually is only constant when all other parameters are fixed. It will vary and be re-computed when there is variation in other parameters and this automatic updating is executed in the syntax programming of MATLAB and also the VIM code, VIMSIM, discussed in due course). Furthermore, there are is the density ratio, $\frac{\widehat{\rho}_{n f}}{\widehat{\rho}_{f}}$, arising in all three momentum and energy conservation Eqns. (32) - (35). The denominator is known from Table 1 (i.e. base fluid density). The numerator is computed from the relation given earlier, namely $\hat{\rho}_{n f}=(1-\phi) \hat{\rho}_{f}+\phi \hat{\rho}_{s}$. Solution of Eqns. (32) - (35) provides 
fundamental primitive dependent variable characteristics i.e. tangential and normal velocity and temperature distributions in the nano-polymer stagnation-point regime. However in materials synthesis, gradients of these functions are also of interest since they have a profound influence on the constitution of final products in polymeric processing [50]. Key gradients are the wall shear stress (normal velocity gradient) and local heat flux (a function of the temperature gradient and radiative flux contribution. These are defined, respectively as follows:

$$
\begin{gathered}
\widehat{\tau}^{*}{ }_{\mathrm{w}}=\left[\mu_{\mathrm{nf}}\left(\widehat{\mathrm{T}}^{*}\right) \frac{\partial \widehat{\mathrm{u}}^{*}}{\partial \widehat{\mathrm{y}}^{*}}\right]_{\widehat{\mathrm{y}}^{*}=0} \\
\widehat{\mathrm{Q}}_{\mathrm{w}}^{*}=-\widehat{\mathrm{k}}_{\mathrm{nf}}\left(\frac{\partial \widehat{\mathrm{T}}^{*}}{\partial \widehat{\mathrm{y}}^{*}}\right)_{\widehat{\mathrm{y}}^{*}=0}+\left(\widehat{\mathrm{q}}_{\mathrm{r}}^{*}\right)_{\widehat{\mathrm{y}}^{*}=0} .
\end{gathered}
$$

In dimensionless form these functions become:

$$
\begin{gathered}
\hat{\tau}_{\mathrm{w}}=\frac{\mathrm{e}^{-\mathrm{m} \theta(0)}}{(1-\phi)^{2.5}}\left(\hat{\mathrm{x}} \mathrm{F}^{\prime \prime}(0)+\gamma \mathrm{H}^{\prime}(0)\right) \\
\widehat{\mathrm{Q}}_{\mathrm{w}}=-\left(\frac{\widehat{\mathrm{k}}_{\mathrm{nf}}}{\widehat{\mathrm{k}}_{\mathrm{f}}}+\frac{4}{3} \mathrm{Rd}\right) \theta^{\prime}(0) .
\end{gathered}
$$

In the MATLAB and VIMSIM computations, dimensionless local heat flux, $\widehat{\mathrm{Q}}_{\mathrm{W}}$, requires values for the thermal conductivity ratio, $\frac{\hat{k}_{n f}}{\hat{k}_{f}}$. Clearly from Eqn. (7), $\frac{\hat{k}_{n f}}{\hat{k}_{f}}=$ $\frac{\hat{k}_{s}+2 \hat{k}_{f}-2 \phi\left(\hat{k}_{f}-\hat{k}_{S}\right)}{\widehat{k}_{s}+2 \widehat{k}_{f}+\phi\left(\hat{k}_{f}-\widehat{k}_{s}\right)}$, the numerator must be evaluated first since the denominator is already prescribed in Table 1. By simple substitution of the volume fraction (first column of Table 2) into Eqn. (7) we readily obtain the values given in the second column of Table 2.

\begin{tabular}{|c|c|}
\hline $\boldsymbol{\phi}$ & $\frac{\hat{k}_{n f}}{\hat{k}_{f}}$ \\
\hline 0 & 1 \\
\hline 0.05 & 1.157133128 \\
\hline 0.1 & 1.331636623 \\
\hline 0.2 & 1.745707932 \\
\hline
\end{tabular}

Table. 2: Thermal conductivity of nanofluid against $\phi$ with $\hat{k}_{f}=0.613, \hat{k}_{s}=400$.

\section{NUMERICAL SOLUTION WITH MATLAB R-K-F QUADRATURE}

The transformed ordinary differential boundary layer equation system is strongly non-linear. Analytical solutions are intractable. Recourse is therefore made to a numerical scheme. Many 
such methods are appropriate for the present stagnation-flow nano-polymer regime including finite element methods, homotopy methods, variational iterative methods (VIMs) and symbolic code quadrature. The last of these methods i.e. numerical quadrature includes numerous variants of which the Runge-Kutta-Fehlberg method of order four [51] is one of the most reliable and accurate when used with a shooting scheme. Here the shooting parameters $b_{1}, b_{2}$ and $b_{3}$ will be calculated by the Newton-Raphson iterative method. Stability and convergence aspects have been lucidly reviewed by Keller [52]. The boundary value problem is converted to an initial value problem and then solved. Let us introduce the following substitutions in Eqs. (32) - (35):

$\mathrm{F}=\mathrm{y}_{1}, \mathrm{~F}^{\prime}=\mathrm{y}_{1}^{\prime}=\mathrm{y}_{2}, \mathrm{~F}^{\prime \prime}=\mathrm{y}_{2}^{\prime}=\mathrm{y}_{3}, \mathrm{H}=\mathrm{y}_{4}, \mathrm{H}^{\prime}=\mathrm{y}_{4}^{\prime}=\mathrm{y}_{5}, \theta=\mathrm{y}_{6}, \theta^{\prime}=\mathrm{y}_{6}^{\prime}=\mathrm{y}_{7},(40)$

Here for the present problem we have :

$\mathrm{y}_{3}^{\prime}=\mathrm{my}_{7} \mathrm{y}_{3}-\xi \mathrm{e}^{\mathrm{my}}{ }_{6}\left(\mathrm{y}_{1} \mathrm{y}_{3}-\mathrm{y}_{2}^{2}+\left(\frac{\mathrm{a}}{\mathrm{c}}\right)^{2}\right)$,

$\mathrm{y}^{\prime}{ }_{5}=\mathrm{my}_{7} \mathrm{y}_{5}-\xi \mathrm{e}^{\mathrm{my}}{ }_{6}\left(\mathrm{y}_{1} \mathrm{y}_{5}-\mathrm{y}_{2} \mathrm{y}_{4}-\mathrm{A}\right)$

$\mathrm{y}_{7}^{\prime}=-\frac{\operatorname{Pr}}{\left(\frac{\widehat{k}_{\mathrm{nf}}}{\mathrm{k}_{\mathrm{f}}}+\frac{4}{3} \mathrm{Rd}\right)}\left(1-\phi+\frac{\left(\hat{\rho} \mathrm{c}_{\hat{\mathrm{p}}}\right)_{\mathrm{s}}}{\left(\widehat{\rho} \mathrm{c}_{\hat{\mathrm{p}}}\right)_{\mathrm{f}}} \phi\right) \mathrm{y}_{1} \mathrm{y}_{7}$,

$\left.\begin{array}{c}y_{1}(0)=0, y_{2}(0)=1, y_{3}(0)=b_{1}, y_{4}(0)=0, y_{5}(0)=b_{2} \\ y_{6}(0)=1+\alpha b_{3}, y_{7}(0)=b_{3}\end{array}\right\}$.

Where $\xi=\left(1-\phi+\frac{\rho_{\mathrm{s}}}{\rho_{\mathrm{f}}} \phi\right)(1-\phi)^{2.5}$.

Computations are executed in Matlab software via the appropriate subroutine.

\section{VALIDATION WITH VARIATIONAL ITERATIVE METHOD (VIM)}

Benchmarking with simpler versions of the general model to published results in the literature achieves only limited validation. In order to validate the general model which includes all the physical parameters i.e. Eqns. (32)-(35), an alternative algorithm must be used to solve the entire boundary value problem. We elect here to employ a powerful and very versatile and easily coded semi-computational method known as the variational iteration method (VIM). Introduced by He [53], the VIM technique can be employed in a direct way without using linearization, perturbation or restrictive assumptions. VIM avoids sensitivity to the degree of 
the nonlinear terms arising in nonlinear polymer processing multi-physical fluid dynamics (and general engineering) problems and also drastically decreases the size of calculations compared with other methods. This method has been deployed very efficiently to resolve numerous nonlinear engineering science problems over the past decade including radiative heat transfer [54], thermoelastic burn injury simulations [55], rocket gel propulsion thermofluid dynamics [56] and ultrasonic heat transfer in biological tissue [57]. VIM is infact a special case of the more general homotopy analysis method. This wide spectrum of applications confirms the exceptional versatility of VIM in modern nonlinear transport phenomena. It is therefore ideal also for nanofluid rheological flows. Here we provide a brief perspective of the technique. Consider the following nonlinear differential equation:

$$
L f(x, y, t)+R f(x, y, t)+N f(x, y, t)=g(x, y, t)
$$

Using VIM, to solve the ordinary differential Eqns. (32) - (34) with boundary conditions (35), we denote here $L f(x, y, t)$ and $R f(x, y, t)$ as linear operators, $N f(x, y, t)$ as the nonlinear operator and $g(x, y, t)$ as He's heterogenous term. VIM uses a correction functional. A successive approximation is established by determining a general Lagrangian multiplier, which can be identified optimally via the variational theory. The first step of employing VIM is to establish the Lagrange multiplier, $\lambda$, which is achieved optimally via integration by parts. The successive approximations of the VIM solution can thereafter be readily obtained via judicious selection of the Lagrange multiplier and by using any selective function, after which several approximations will naturally follow immediately. The correctional assumes the form:

$$
\delta f_{n+1}(x, y, t)=\delta f_{n}(x, y, t)+\delta \int_{0}^{t} \lambda\left\{L f_{n}(x, y, t)+R f_{n}(\hat{x}, y, t)+N f_{n}(\hat{x}, y, t)\right\} d \tau
$$

Here $\lambda$ is general Lagrange multiplier which can be identified optimally via variational theory, $\hat{R} f_{n}$ and $\hat{N} f_{n}$ are considered as restricted variations i.e.

$$
\delta \hat{R} f_{n}=0, \delta \hat{N} f_{n}=0
$$

Algebraic details are omitted for brevity. The computations are executed in Mathematica symbolic software on a HP Workstation and take tens of seconds to converge. Selected values for nano-polymer thermal conductivity ratio for different nano-particle volume fractions are presented in Table 2. 


\begin{tabular}{|c|c|c|c|c|c|c|}
\hline Rd & $\begin{array}{c}\text { R-K-F } \\
\text { Quadrature }\end{array}$ & VIM & $\begin{array}{c}\text { R-K-F } \\
\text { Quadrature }\end{array}$ & VIM & $\begin{array}{c}\text { R-K-F } \\
\text { Quadrature }\end{array}$ & VIM \\
& $\boldsymbol{\phi}=\mathbf{0}$ & $\boldsymbol{\phi}=\mathbf{0}$ & $\boldsymbol{\phi}=\mathbf{0 . 0 5}$ & $\boldsymbol{\phi}=\mathbf{0 . 0 5}$ & $\boldsymbol{\phi}=\mathbf{0 . 1}$ & $\boldsymbol{\phi = 0 . 1}$ \\
\hline 0 & 1.505504 & 1.505601 & 1.597741 & 1.597698 & 1.693085 & 1.693102 \\
\hline 5 & 2.959719 & 2.959432 & 2.890633 & 2.890594 & 2.861669 & 2.861684 \\
\hline 9 & 3.524343 & 3.523998 & 3.408865 & 3.409032 & 3.349474 & 3.349489 \\
\hline
\end{tabular}

Table. 3: Shear stress for various $R d$ and $\phi$ with $\frac{a}{c}=0.1, m=0.1, \alpha=0.1$.

\begin{tabular}{|c|c|c|c|c|c|c|}
\hline Rd & $\begin{array}{c}\text { R-K-F } \\
\text { Quadrature }\end{array}$ & VIM & $\begin{array}{c}\text { R-K-F } \\
\text { Quadrature }\end{array}$ & VIM & $\begin{array}{c}\text { R-K-F } \\
\text { Quadrature }\end{array}$ & VIM \\
& $\boldsymbol{\phi}=\mathbf{0}$ & $\boldsymbol{\phi}=\mathbf{0}$ & $\boldsymbol{\phi}=\mathbf{0 . 0 5}$ & $\boldsymbol{\phi}=\mathbf{0 . 0 5}$ & $\boldsymbol{\phi = 0 . 1}$ & $\boldsymbol{\phi = 0 . 1}$ \\
\hline 2 & 2.295769 & 2.295688 & 2.292533 & 2.292469 & 2.311067 & 2.311091 \\
\hline 4 & 2.773922 & 2.774011 & 2.722204 & 2.722197 & 2.705276 & 2.705301 \\
\hline
\end{tabular}

Table. 4: Local heat flux against $R d$ with $\frac{a}{c}=0.1, m=0.1, \alpha=0.1$.

The comparisons for numerical quadrature (Matlab) and VIM are documented in Table 3 and Table 4 for dimensionless shear stress and local heat flux with various radiation-conduction parameters $(R d)$ and nano-particle volume fractions $(\phi)$ and with secondary stretching ratio i.e. obliqueness parameter $(\gamma)$ prescribed as 0.1 , Reynolds viscosity exponent $(m)$ fixed as 0.1 and thermal slip parameter $(\alpha)$ constrained as 0.1 . Excellent correlation is achieved. Confidence in the numerical quadrature solutions (R-F-K MATLAB code) is therefore justifiably high.

\section{RESULTS AND DISCUSSION}

Copper nano-particles doped polymers are considered i.e. in which copper nano-particles are added in water-based polymer (sol gel). Figs. (2) - (7) are plotted to explore the influence of solid volume fraction of nanoparticles $\phi$, radiation parameter $R d$ and thermal slip parameter $\alpha$ on velocity and temperature profiles. Fig (8) depicts a histogram of shear stress versus nanoparticles volume fraction $\phi$ for various values of radiation parameter $R d$. Local heat flux $-\left(\frac{\widehat{\mathrm{k}}_{\mathrm{nf}}}{\widehat{\mathrm{k}}_{\mathrm{f}}}+\frac{4}{3} \mathrm{Rd}\right) \theta^{\prime}(0)$ for numerous values of radiation parameter $R d$ is presented in the histogram in Fig. (9). Two-dimensional streamlines with obliqueness parameter (secondary stretching and contracting ratio) values of $\gamma=10$ and $\gamma=-10$ for radiation parameter and thermal slip parameter are presented in Figs. (10) - (13). We note that in Figs 2, 3, 5 we have also further validated the R-K-F MATLAB solutions with the VIM code for at least one profile (designated with the logo ). Again, it is evident that excellent agreement is achieved. 


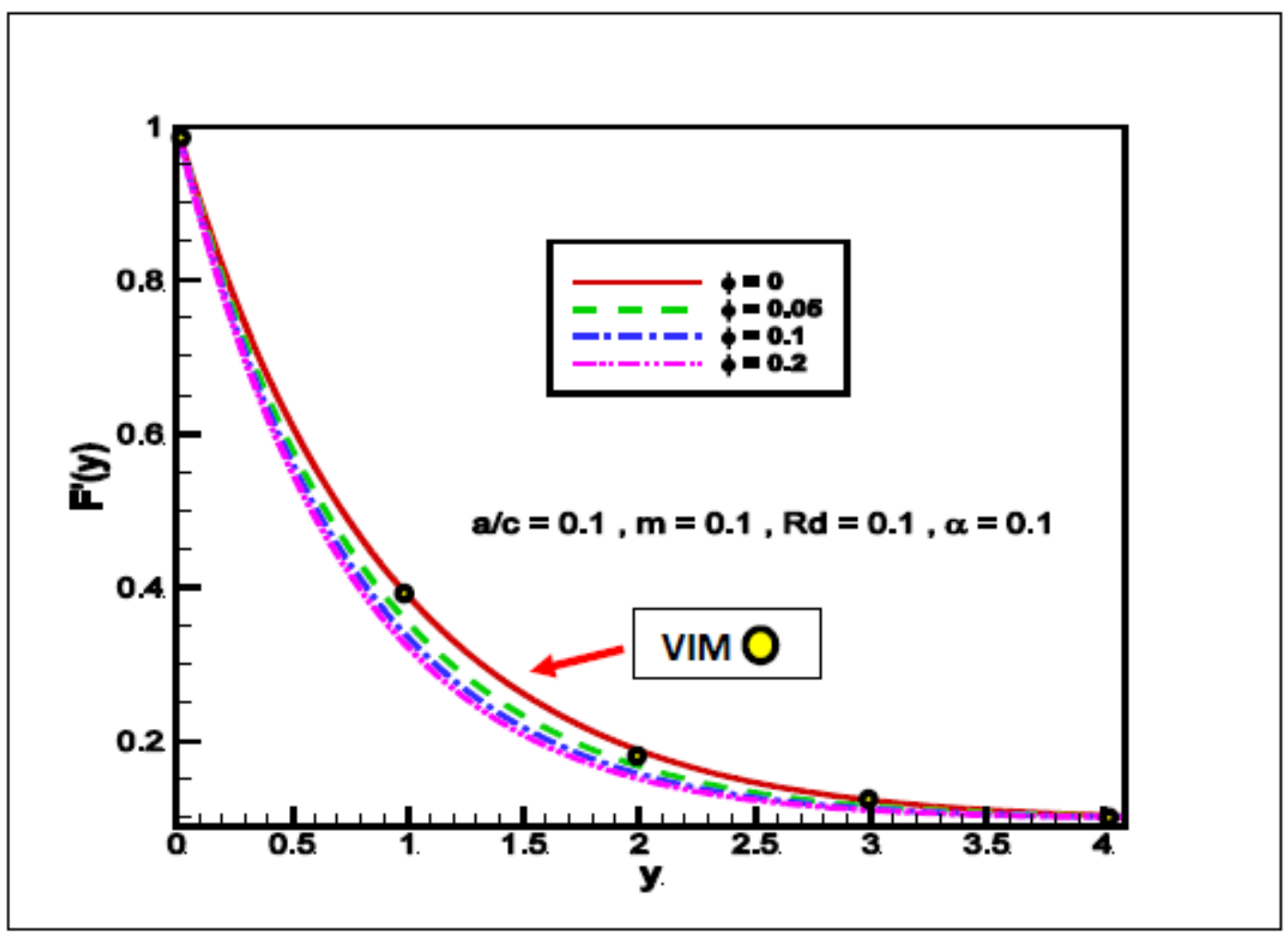

Fig 2. Normal velocity profile $F^{\prime}(y)$ with nano-particle volume fraction $(\phi)$.

Fig. (2) exhibits that normal velocity profile $\mathrm{F}^{\prime}(\mathrm{y})$ decreases with increase in $\mathrm{Cu}$ nanoparticles volume fraction. The presence of nano-particles alters the viscosity of the sol-gel. Increasing rheological effects are generated with greater volume fraction, as corroborated experimentally by Chen et al. [58]. The shear-thinning behaviour of nano-sol gels is strongly influenced by the effective particle concentration, the range of shear rate and viscosity of the base liquid. Greater viscosity generates higher viscous resistance forces which manifest in deceleration in the stagnation flow. Greater flow control in the stagnation manufacturing regime is therefore achieved comfortably with increased doping with copper nano-particles. The enhanced thermal conductivity of the nano-polymer also means that heat is diffused faster than momentum. The ratio of momentum and thermal diffusivities is expressed via the Prandtl number which is fixed when these two diffusivities are fixed. Prandtl number however is also proportional inversely to thermal conductivity for a prescribed dynamic viscosity and specific heat capacity (isobaric). Most water-based sol gels even with low nano-particle doping will have approximately a Prandtl number of 6-8. However, this will be decreased considerably with increasing thermal conductivity as the nano-particle volume fraction is increased. In fact, nano-polymers may be categorized in four groups depending on the extent of volume fraction i.e. dilute, semi-dilute, semi-concentrated and concentrated nanofluids depending on particle concentration and 
particle structuring, although in our analysis we have assumed dilute suspensions in thermal equilibrium. Enhanced nano-doping clearly will result in faster conduction of thermal energy in the nano-polymer and slower vorticity diffusion and therefore retardation in the flow i.e. decreasing velocities. In all the profiles asymptotic smooth decays are computed from the wall (solid/nano-polymer interface) to the free stream outside the stagnation zone. This confirms the imposition of an adequately large infinity boundary condition in the free stream (edge of the boundary layer) verifying that the numerical solutions exhibit indeed the correct nature. Maximum influence of the nano-particle volume fraction arises at intermediate distance from the wall since here the nano-particles can cluster in greater concentrations. The influence is consistent on velocity (i.e. deceleration) throughout the stagnation-flow boundary layer regime, concurring with the experimental and theoretical findings of Wedgewood and Joshi [59]. It is however not-worthy that since steady state flow has been considered in the current model, the time-dependent phenomena cannot be captured. Transient models reveal that in the stagnation region the nano-polymer is not completely stagnant but follows a non-streamwise motion. These aspects will be studied in the future as they are beyond the scope of the current work. Nevertheless, the present model still retains validity in actual coating flows and generalizes the conventional orthogonal case (featuring only a normal impingement velocity) to the industrially relevant scenario of oblique flow, as elaborated by Lee and Tallmadge [60].

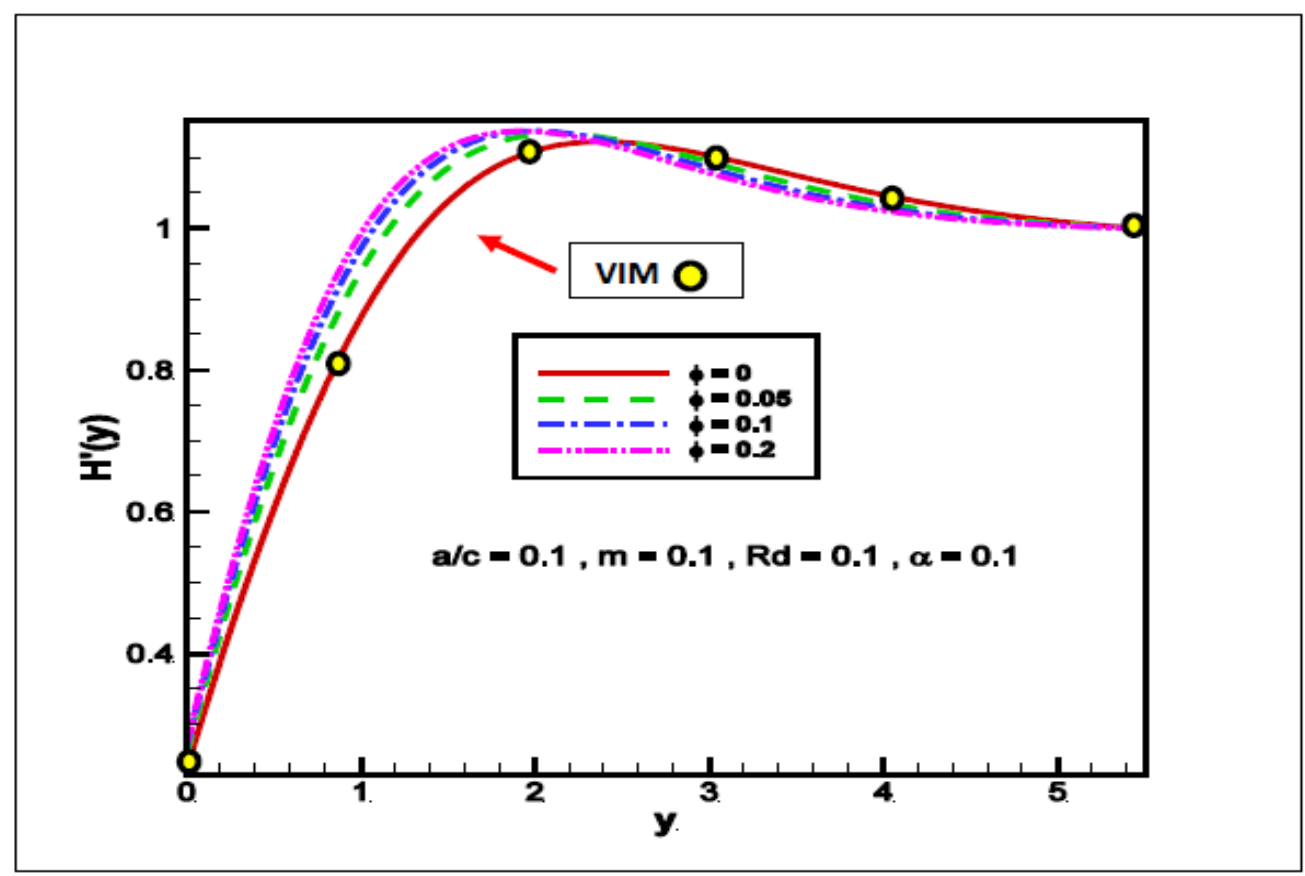

Fig. (3): Oblique (tangential) velocity profile $H^{\prime}(y)$ with nanoparticles volume fraction $(\phi)$. 


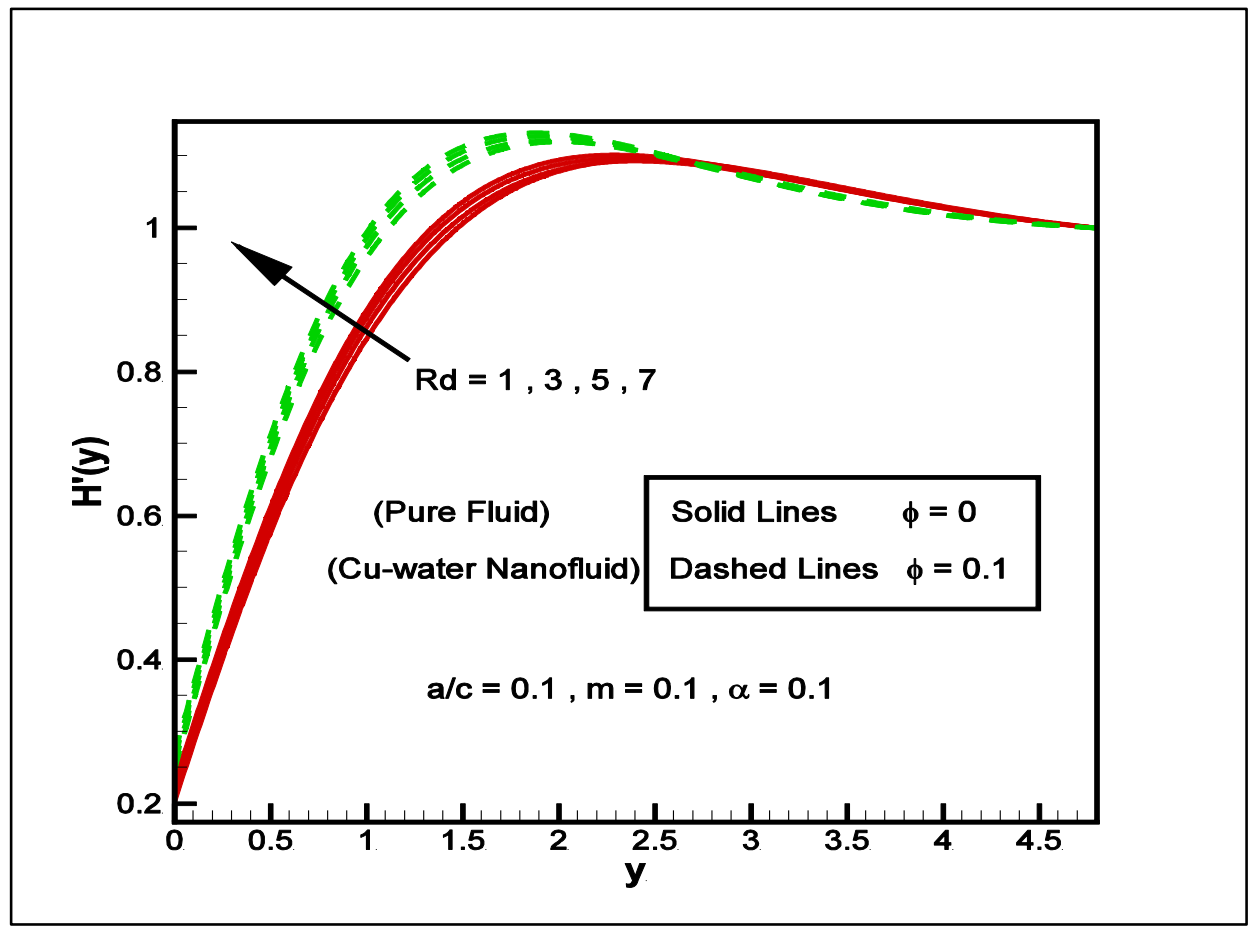

Fig. (4): Tangential velocity profile $H^{\prime}(y)$ with various conduction-radiation parameters $\left(R_{\mathrm{d}}\right)$.

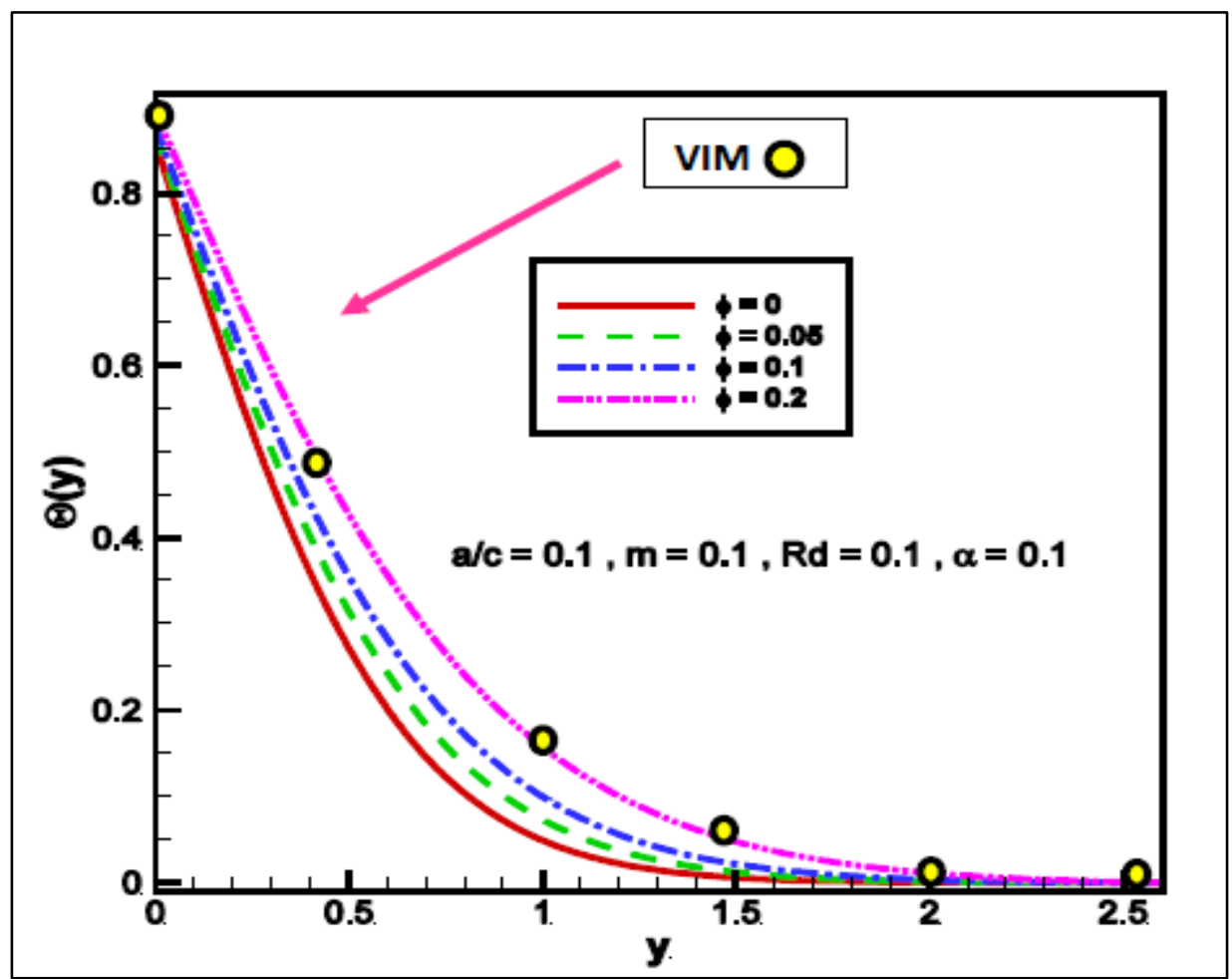

Fig. (5): Temperature profiles, $\theta(y)$, with variation in nanoparticle volume fraction $(\phi)$. 


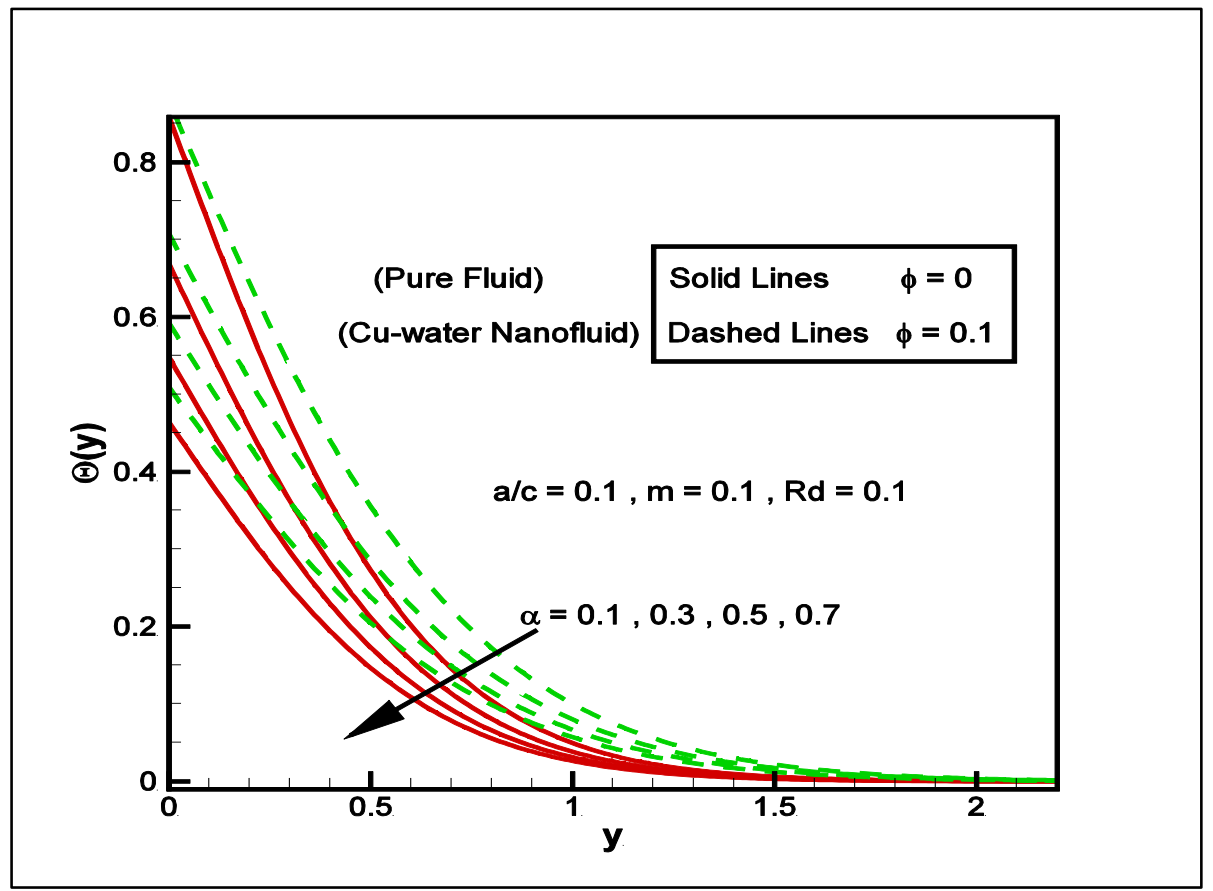

Fig. (6): Temperature profiles, $\theta(y)$, for various thermal slip parameters $(\alpha)$.

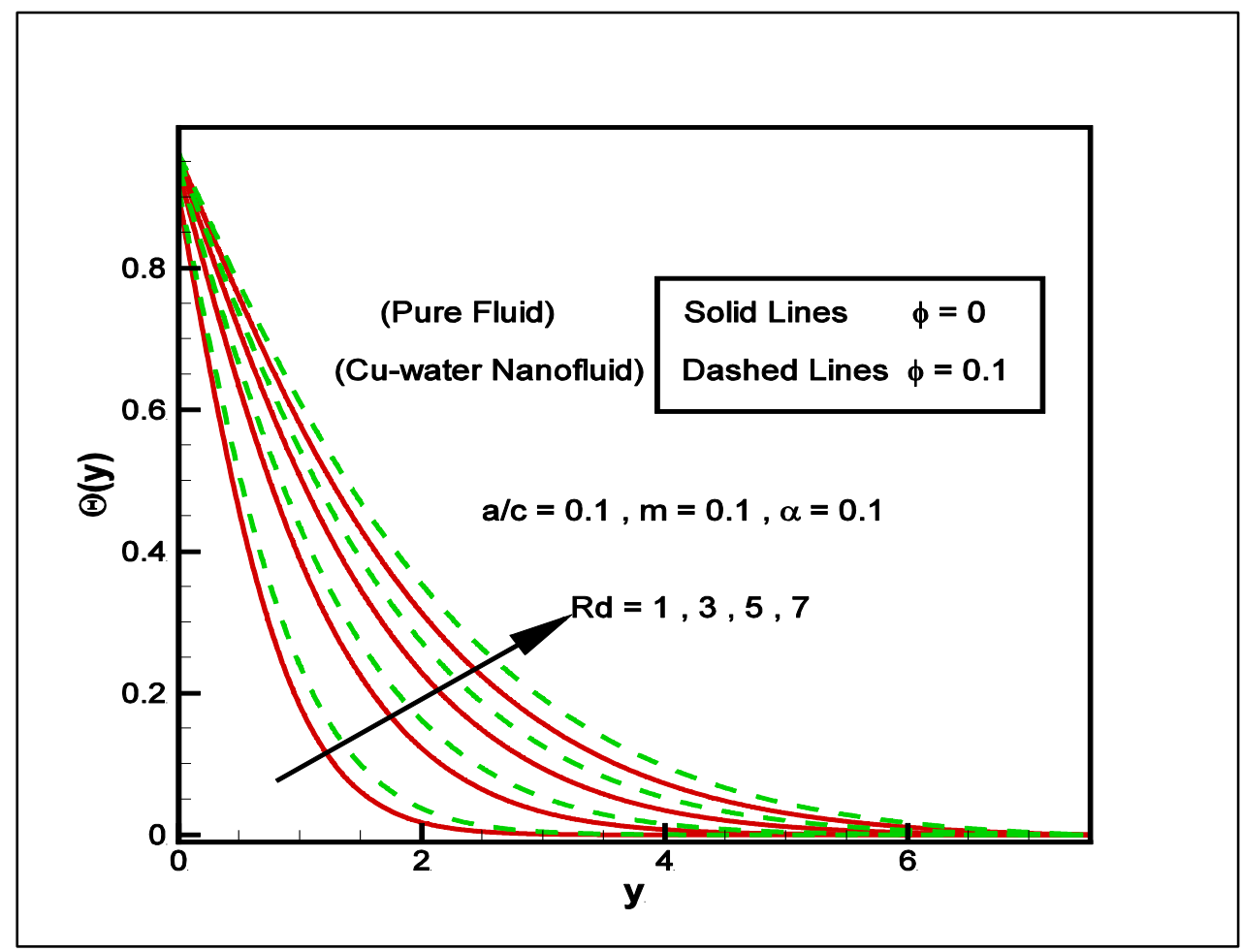

Fig. (7): Temperature profiles, $\theta(y)$, with various conduction-radiation parameters $(R d)$. 


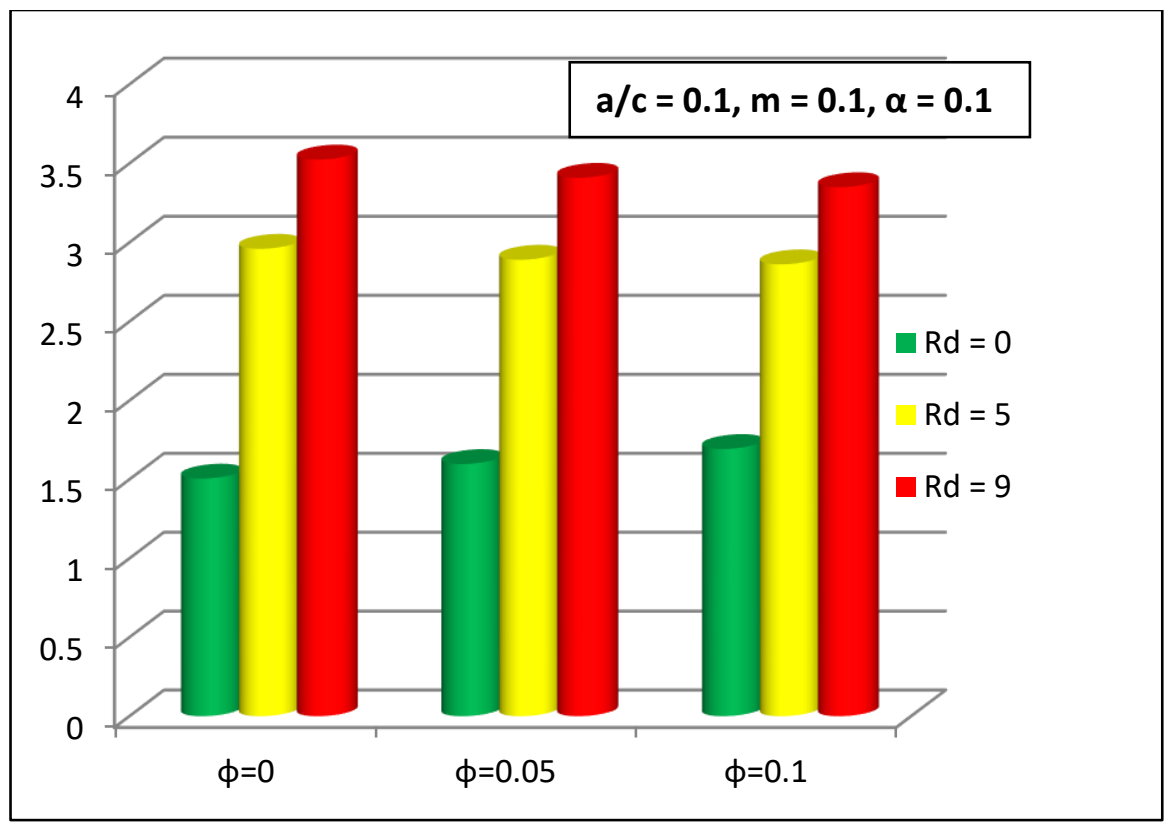

Fig. (8): Shear stress $\frac{\mathrm{e}^{-\mathrm{m} \theta(0)}}{(1-\phi)^{2.5}}\left(\mathrm{~F}^{\prime \prime}(0)+\gamma \mathrm{H}^{\prime}(0)\right)$ for various nano-particle volume fractions $(\phi)$ and conduction-radiation parameter values $(R d)$ at $\hat{x}=1$.

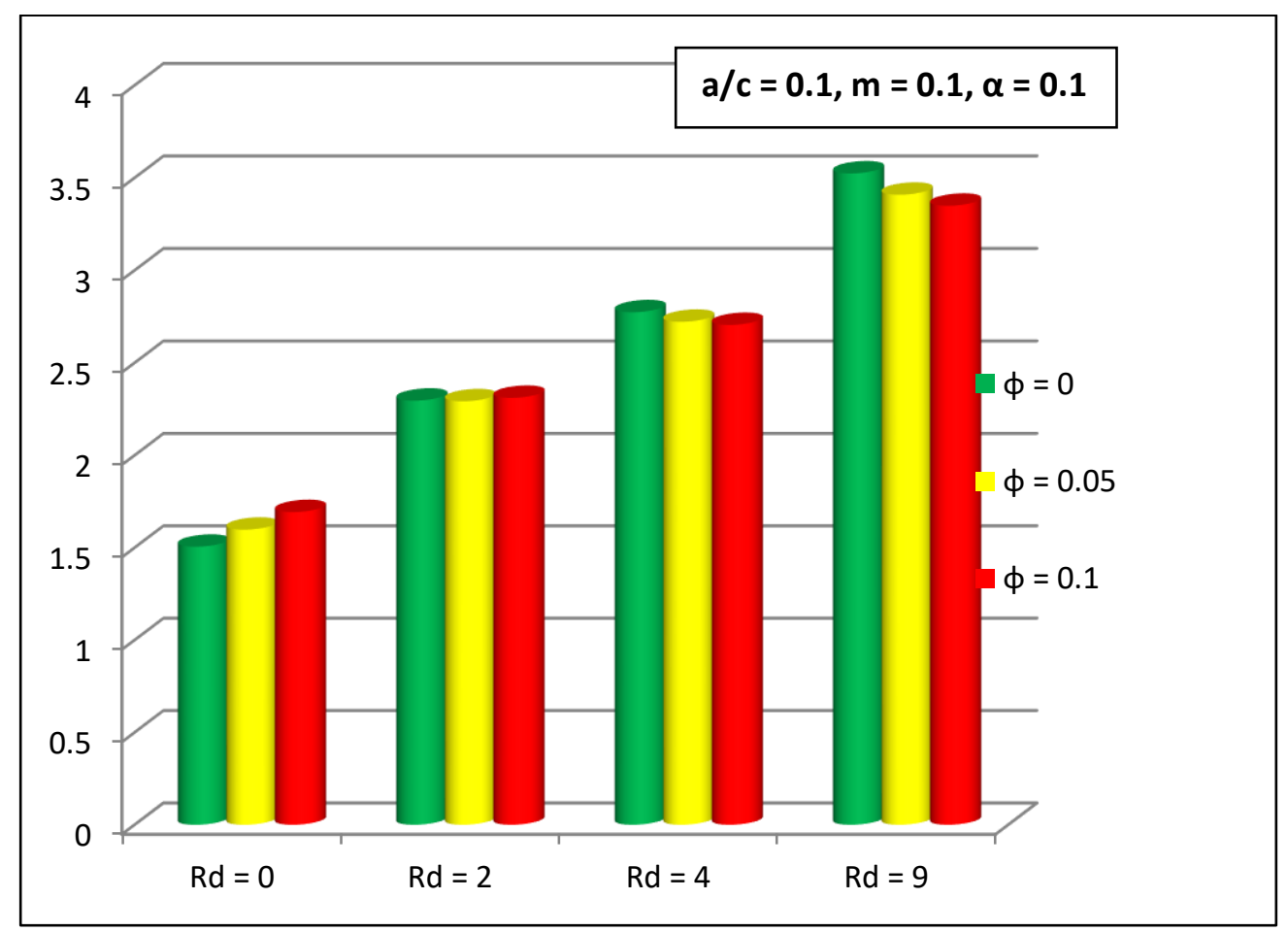

Fig. (9): Local heat flux $-\left(\frac{\widehat{\mathrm{k}}_{\mathrm{nf}}}{\widehat{\mathrm{k}}_{\mathrm{f}}}+\frac{4}{3} \mathrm{Rd}\right) \theta^{\prime}(0)$ against nano-particle volume fraction $\phi$. 


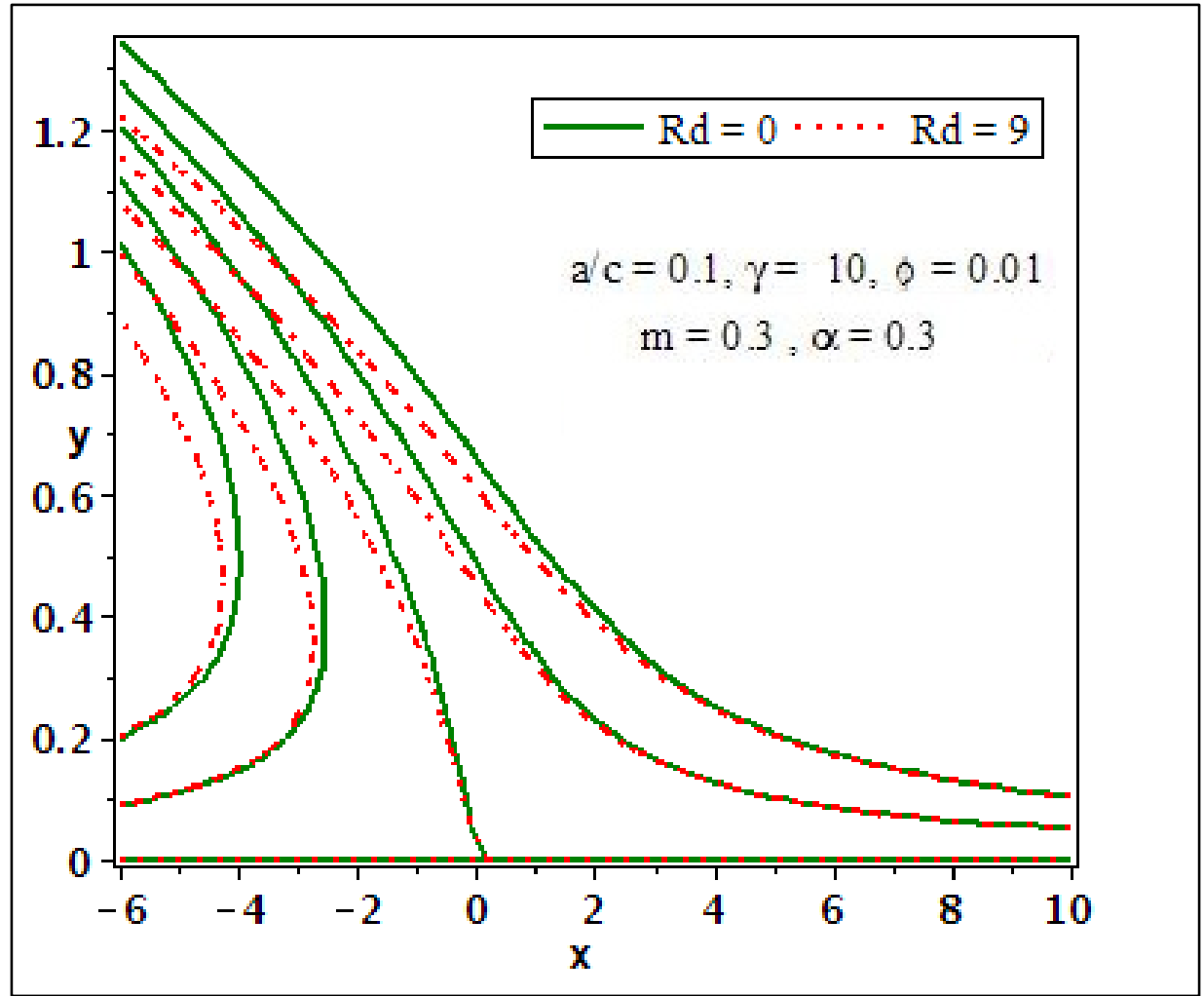

Fig. (10): Streamlines for conduction-radiation parameters $(R d)$ with obliqueness $(\gamma)=10$.

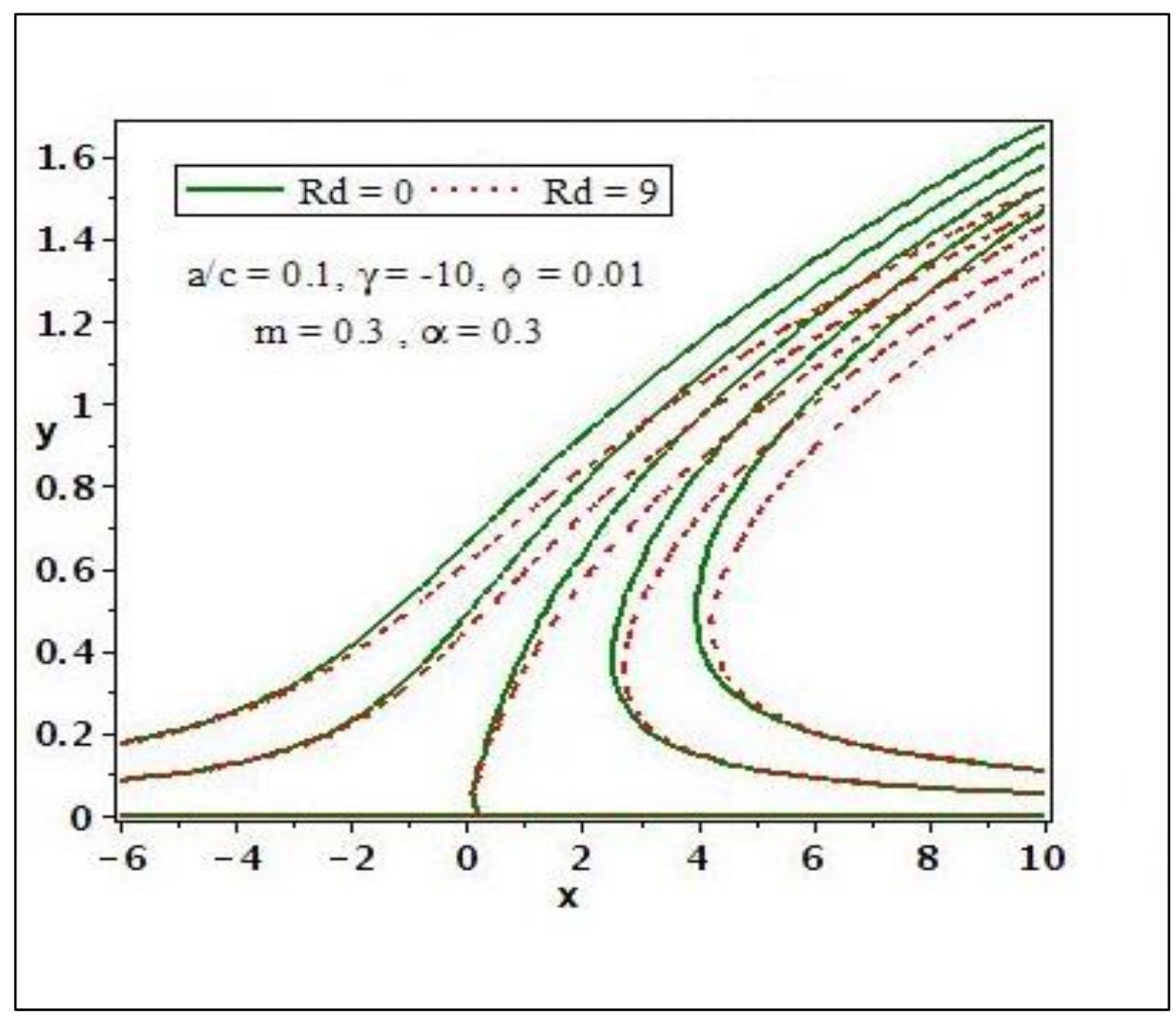

Fig. (11): Streamlines for conduction-radiation parameters $(R d)$ with obliqueness $(\gamma)=-10$. 


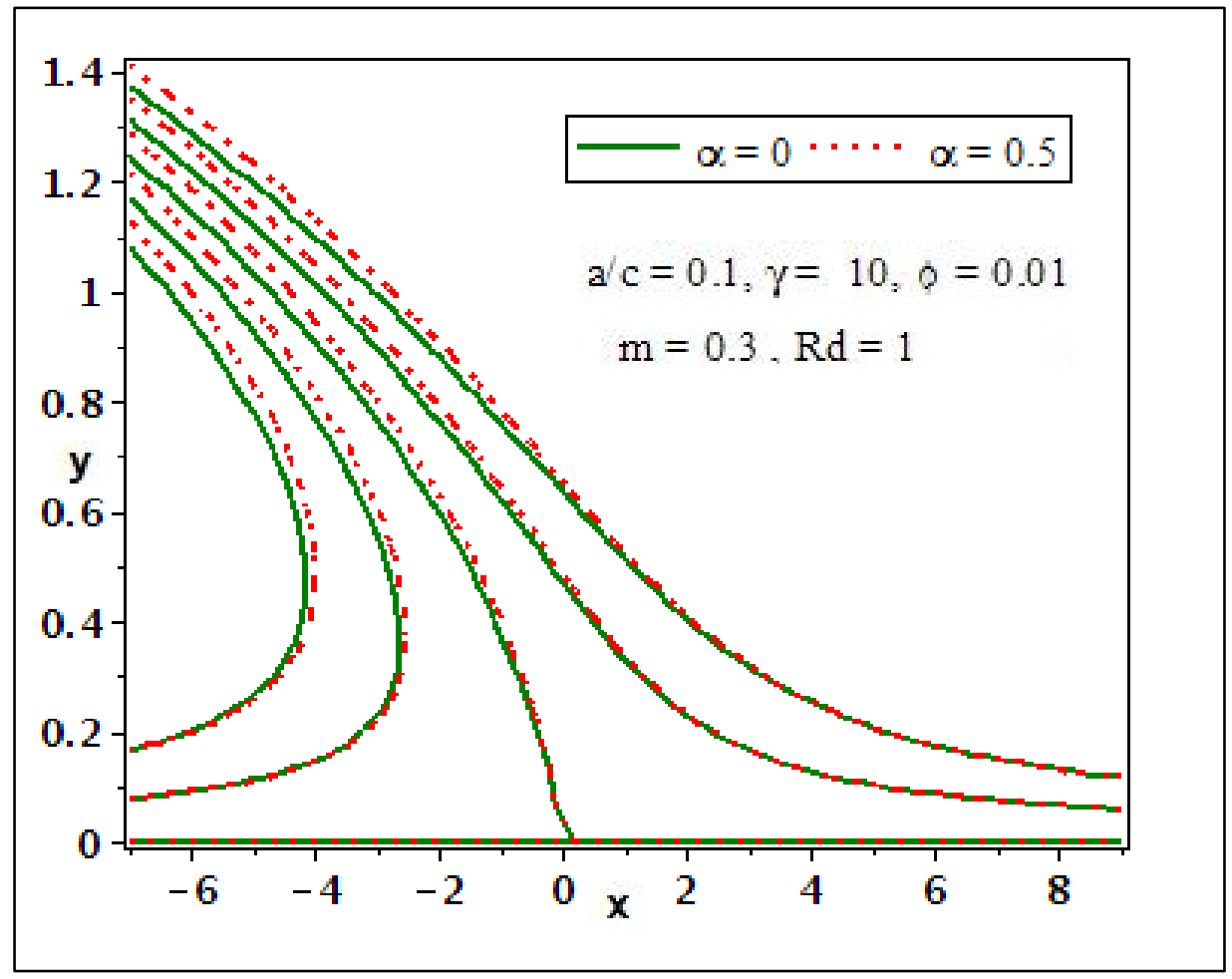

Fig. (12): Streamlines for thermal slip parameters $(\alpha)$ with obliqueness $(\gamma)=10$.

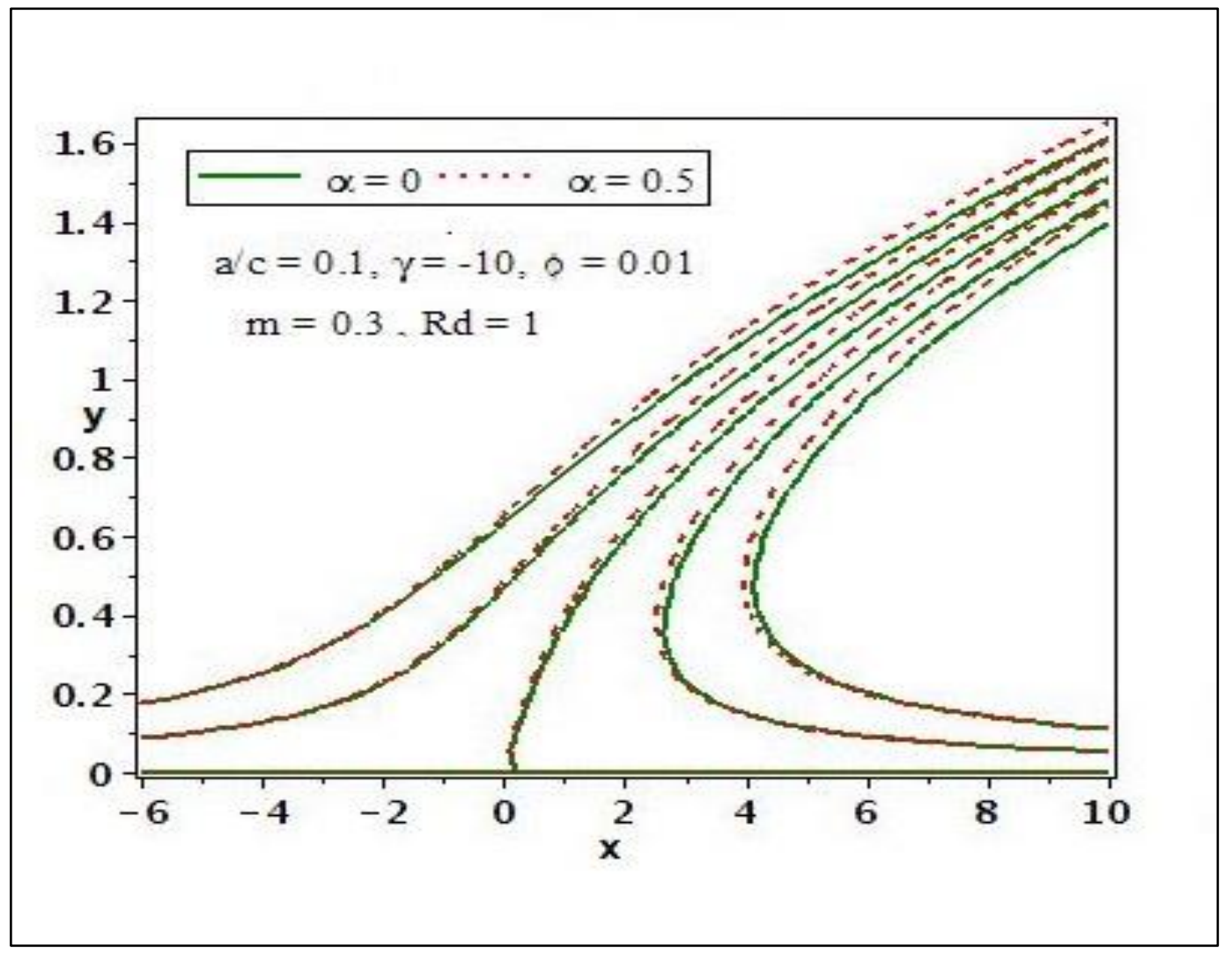

Fig. (13): Streamlines for thermal slip parameter $\alpha$ with $\gamma=-10$. 
Fig. (3) shows that the oblique velocity component (tangential) velocity profile $H^{\prime}(y)$ near the surface is enhanced by increasing copper nanoparticle volume fraction; however deeper into the boundary layer transverse to the solid surface, there is a reversal in this effect. This is intimately associated with the re-distribution in the dual momentum field, analogous to some extent to that encountered in Von Karman swirling flows. The destruction in normal momentum is compensated for with an accentuation in tangential momentum in particular near the solid surface. The distinction is however that while the depletion in normal momentum (Fig. 2) is consistent everywhere in the boundary layer with increasing nano-particle volume fraction, in the tangential momentum the corresponding accentuation is only localized in the lower structure of the boundary layer. As we proceed to the free stream, the external effects dominate and reverse the tangential momentum escalation encountered earlier. Effectively there is a sustained increase in normal momentum boundary layer thickness whereas there is a dual response in the tangential momentum boundary layer thickness which is initially thinner (for acceleration near the wall) and thicker subsequently (for deceleration towards the free stream). The stream function infact splits into a Hiemenz and a tangential component. Furthermore, it is pertinent to mention that while a stability analysis could be conducted for the stagnation flow, this has already been done for similar regimes by a number of other workers. Notable among these studies is the analysis of Paullet and Weidman [61] and Mahapatra et al. [62] (although attention was restricted to purely viscous Newtonian fluids in these two studies) and later Lok et al. [63] who considered micropolar fluids, not nanofluids. Generally, in these studies it has been shown that multiple solutions can be derived for non-orthogonal stagnation flows. For primary (normal) sheet stretching ratio, $a / c>1$, the range of solutions is lower than the stable solutions (i.e. it is encompassed in the stable solution range) due to an increase in a/c whereas for $\mathrm{a} / \mathrm{c}<1$ the range of unstable solution become larger than the stable solution. They found that first solution is applicable physically while the second solution is not. Since we are concerned with physically realizable nano-polymer processing in this study, as engineering scientists, we restrict our attention to the second family of solutions within which the present computations fall. In the case studied $(\mathrm{a} / \mathrm{c}<1)$ the correct physical behaviour i.e. vorticity external to the boundary layer is present, not within the boundary layer.

Fig. (4) exhibits the evolution in tangential velocity profile $H^{\prime}(y)$ for both cases of pure waterbased polymer and also copper $(\mathrm{Cu})$-water nano-polymer fluid with variation in conductionradiation parameter $(R d)$. It is evident that tangential velocity profile of copper $(\mathrm{Cu})$-water nano-polymer exceeds markedly in magnitude than that of the pure water-based polymer fluid. 
However further from the solid surface the converse behavior is computed i.e. deceleration is induced with nano-particle doping of the polymer towards the free stream. The figure also clearly demonstrates that increasing the conduction-radiation parameter $R d$ generally enhances the tangential velocity $H^{\prime}(y)$ magnitudes. Radiative heat transfer is crucial when the radiant heat flux, is large compared to the heat transfer rate due to convection or conduction. Typically, this will occur under high-temperature conditions, associated with nano-polymer synthesis in solar coatings [64] for which the fourth-order dependence of the radiative heat flux on temperature implies that the radiation heat transfer will dominate. The parameter, $R d=\frac{4 \widehat{\delta}^{*} T_{\infty}^{3}}{\widehat{k}_{f} K^{*}}$, quantifies the importance of conduction versus radiation within the fluid. The parameter $R d$ arises in the augmented energy Eqn. (35) i.e. in the term $\left(\frac{\widehat{\underline{n}}_{\mathrm{nf}}}{\widehat{\mathrm{k}}_{\mathrm{f}}}+\frac{4}{3} \mathrm{Rd}\right) \theta^{\prime \prime}$. Rosseland's model assumes radiative equilibrium and that the nanofluid has gray properties which are physically viable in nano-polymer sol gel processing under uni-directional radiative thermal loading as described by Said et al. [65]. Furthermore, Rosseland's model assumes that the intensity is the black-body intensity at the nano-polymer temperature and since it is generally confined to incompressible flows it is particularly appropriate for viscous-dominated manufacturing processes. Conductive heat transfer dominance is ensured in the thermal boundary layer, when $R d \rightarrow 0$ i.e. the radiative contribution vanishes. With $R d>0$ thermal radiation influences the regime i.e. a progressively greater quantity of radiation heat transfer is received within the nano-polymer which results in energization of the fluid and a rise in temperatures. This behaviour is indeed captured in Fig. 7 (described later). The energization of the flow regime will serve to enhance thermal diffusion and therefore elevates temperatures and also thermal boundary layer thickness. This will deplete the primary (normal) momentum field i.e. decelerate the normal velocity component but will enhance the secondary (tangential) velocity component manifesting in the acceleration observed in Fig. 4, again largely in the near-wall region of the boundary layer. Significant manipulation of the dual momentum fields in the oblique stagnation nano-polymer flow regime is therefore attained with the imposition of radiative heat transfer.

Fig. (5) presents the distributions in nano-polymer temperatures with an increase in copper nanoparticle volume fraction $\phi$. The trend confirms the principal benefit of doping with nanoparticles, namely the enhancement in nano-material thermal conductivity which results in a exacerbated thermal conduction and a boost in temperatures. There will also be a corresponding elevation in thermal boundary layer thickness. Although the Tiwari-Das model studied does 
not possess the framework to simulate advanced nanoscale phenomena e.g. thermophoresis and Brownian motion, these do nevertheless contribute greatly to the global effect of nano-particle doping, irrespective of the mathematical model employed. Different nanoparticle types transport differently under the thermophoretic force which is associated with migration of nano-particles from hot to cold surfaces. This is of great utility in designing nano-polymers and indeed contributes tremendously to their improved performance in solar coating applications compared with conventional first or second generation solar coatings [66]. Via selection of specific nano-particles, customized performance may be achieved. It is further of interest to consider the mechanism of thermal enhancement in nanofluids under solar radiative conditions. Since Brownian dynamics is inevitably present, the nanofluid molecules are organized into an ordered layer at the nano-particle solid-liquid interface which results in thermal conductivity in that ordered layer being lower than thermal conductivity of the solid nano-particles but larger than that of the base fluid. As recently emphasized by Bég [67] and earlier suggested by Buongiorno [68], this interfacial layer is a solid-like structure, and it is referred to as nanolayer. This hypothetical nanometre size layer is considered as a thermal layer between the solid particle surface and the base fluid and to the current state of knowledge of nanofluid mechanics, this nanolayer is one of the most probable mechanisms producing the popular thermal conductivity enhancement. Infact, the existence of even a thin nanolayer with strong $h$ Brownian motion (likely at even low nano-particle volume fractions) still may contribute markedly to the elevation in thermal conductivity of nanofluids, mainly when the particle diameter is smaller than $10 \mathrm{~nm}$. In conjunction with thermophoresis, the extra energy transport of nanoparticles is due to result of Brownian motion. The relative motion between nanoparticles and base fluid molecules generates micro-convection which when summated over the entire body of the nano-polymer results in an effective boost in heat transfer i.e. temperatures. The present theoretical results confirm these observations. However, it is sincerely hoped that experimental solar engineering researchers will be motivated to verify actual computations with laboratory models and scale-up processes to actual implementation of the current solar nano-polymer model.

Fig. (6) illustrates the response in nano-polymer temperature profile $\theta(y)$ with various values of thermal slip parameter $(\alpha)$ for both pure water polymers and copper-water nano-polymers. Consistently the copper-water nano-polymer achieves significantly higher temperatures than the pure water polymer. With greater thermal slip (jump) there is a strong reduction in temperature and therefore thermal boundary layer thickness. The necessity of including thermal 
slip is both related to actual interfacial solid/nano-polymer effects and also to the shortcomings of the Rosseland model which requires modification with thermal slip boundary conditions. Clearly with weak thermal slip (or indeed in the absence of thermal slip) higher temperatures are computed. The implication is that in models which neglect thermal slip, the temperature generated in nano-polymers is over-predicted and not realistic even with relatively low thermal radiation present $(R d=0.1)$. Thermal slip has a particularly strong impact at the wall, as expected. The influence decays progressively with distance into the boundary layer regime transverse to the solid surface (wall). Temperature distributions eventually merge in the free stream where the temperature field is essentially unaffected by the thermal slip since the latter is a boundary condition and not associated with the mass of the nano-material. Again, very smooth convergent profiles are achieved in the free stream confirming that a sufficiently large infinity boundary condition has been employed in the computations.

Fig. (7) presents the temperature profile variation with a change in conduction-radiation parameter $(R d)$. For $R d>1$ thermal radiation dominates over thermal conduction. When $R d<1$ thermal conduction dominates. When $R d=1$ both thermal conduction and thermal radiation contributions are equal. Increasing $R d$ clearly strongly boosts temperatures since divergence of the radiative heat flux increases and the greater the radiative flux imparts greater thermal energy to the nano-polymer. Thermal boundary layer thickness is therefore also strongly increased. It is also noticed that temperature profile of fluid with copper nanoparticles is substantially higher than pure fluid due to the thermal conductivity strength of nanofluid. Therefore, thinner thermal boundary layers are produced in the stagnation flow regime with lower values of $R d$ since in this case there is a minimal augmentation of the overall thermal diffusivity of the nanopolymer. Similar findings have been reported recently by Uddin et al. [69].

Figs. (8) and (9) present bar charts for the variation in respectively normal shear stress (gradient of normal velocity component at the wall) and heat flux with various values of nanoparticle volume fraction $(\phi)$ and conduction-radiation parameter $(R d)$. Fig. (8) indicates that in the absence of radiation effects $(R d=0)$, primary (normal) shear stress $\frac{\mathrm{e}^{-\mathrm{m} \theta(0)}}{(1-\phi)^{2.5}}\left(\mathrm{~F}^{\prime \prime}(0)+\right.$ $\left.\gamma \mathrm{H}^{\prime}(0)\right)$ increases at the surface with greater $\phi$ values. However, normal shear stress is found to decline with increasing $\phi$ values when radiation effects are incorporated. There is ostensibly therefore a trade-off between the nano-particle doping and intensity of radiative thermal loading. Non-radiative heating leads to acceleration at the wall with greater nano-particle doping whereas strongly radiative heating manifests in a deceleration in the stagnation flow 
normal component. From Fig. (9) it is apparent that an enhancement in local heat flux is generated with increasing conduction-radiation parameter. An important feature computed here is that rate of heat transfer i.e. local heat flux decreases with increasing nano-particle volume fraction $\phi$ when thermal radiation is present $(R d>0)$. However, the converse effect is observed i.e. local heat flux is consistently elevated with increasing nano-particle volume fraction $\phi$ in the absence of thermal radiative heat transfer $(R d=0)$.

Figs.(10)-(13) visualize the two-dimensional streamline distributions with variation in obliqueness (secondary stretching) parameter $(\gamma)$, and conduction-radiation parameter $(R d)$ and thermal slip parameter $(\alpha)$. Clearly there is a strong skewness of the stagnation nano-polymer flow towards the left in Fig. (10) with obliqueness parameter $\gamma=10$. However, with $\gamma=-10$, (Fig. 11) the opposite response is generated and the streamlines are strongly skewed towards the right hand side. Similar computations have been reported by Nadeem et al. [70] although they considered viscoplastic nano-polymers.

With increasing thermal radiation contribution, the streamline magnitudes are noticeably reduced in Fig. 10 and again a similar response is computed in Fig. 10. The obliqueness therefore does not exert any tangible effect on the impact of thermal radiation in these two figures. Fig. (12) shows that streamlines of the nanofluid flow with obliqueness parameter $\gamma=$ 10 and without thermal slip effects are orientated strongly towards the left side when compared with the streamlines of the flow with thermal slip effects. The contrary behaviour is observed in Fig. (13) with $\gamma=-10$. However in both cases there is a slight enhancement in streamline magnitudes with thermal slip parameter. The positive obliqueness is associated with an increase in pressure and straining motion near the stagnation point which results in thinning of velocity boundary layer and thickening of the thermal boundary layer.

\section{CONCLUDING REMARKS}

Thermal slip has been studied in obliquely impinging radiative flow of a copper $(\mathrm{Cu})$-water nano-polymeric solar coating on a stretching surface. Viscosity of the nano-polymer is assumed to be dependent upon temperature and a Reynolds exponential viscosity model is deployed to this effect. A two-dimensional steady state model has been developed for the non-orthogonal stagnation flow with a Rosseland radiative flux model which is valid for gray media. The present study has been motivated by analysing in detail the characteristics of non-aligned nano- 
polymer manufacturing flows. The Tiwari-Das nano-particle volume fraction model has been used in which nano-polymer thermal conductivity, density and specific heat capacity can be computed for different concentrations of nano-particles. The normalized ordinary differential boundary value problem, which is strongly nonlinear and features both normal and tangential momentum equations and a heat (energy) conservation equation has been solved with both Matlab-based Runge-Kutta-Fehlberg numerical quadrature and a variational iteration method (VIM). Very good correlation of the computations has been achieved. The principal findings of our investigation can be summarized as below:

- Temperatures are elevated with increasing volume fraction of copper nanoparticles and increasing thermal radiation effects whereas they are suppressed with increasing thermal slip parameter.

- Temperatures of the copper water nano-polymer are consistently greater in magnitude than those achieved with pure water polymer.

- Shear stress and heat flux are markedly boosted with increasing thermal radiation parameter.

- Streamlines are more tilted towards the left-hand side with thermal radiation present as compared to usual streamline patterns for positive obliqueness parameter. The converse behaviour is computed for negative obliqueness parameter.

- For both positive and negative obliqueness parameter (secondary stretching ratio) However in both cases there is a slight enhancement in streamline magnitudes with thermal slip parameter.

The current investigation has been confined to steady state, two-dimensional oblique flow with a simple non-Newtonian viscosity variation. Future simulations will consider time-dependent effects in three-dimensional oblique nano-polymer flows and will also incorporate more sophisticated non-Newtonian (rheological) models for the nano-polymer e.g. viscoelastic and viscoplastic. The results of these studies which may also utilize computational fluid dynamics codes e.g. ANSYS FLUENT. Furthermore, an important aspect not considered in the current model is the stability of the nano-particles. This is a complex issue which has been addressed notably by Shi et al. [72] for $\mathrm{Fe} 3 \mathrm{O} 4 \mathrm{CNT}$ nanoparticles and again computational fluid dynamics and also experimentation would be good pathways to investigate this.

Conflict of Interest: The authors declare that they have no conflict of interest with any individual or organization regarding this articles content. 


\section{REFERENCES}

[1] J. Liu and S. Bashir, Advanced Nanomaterials and Their Applications in Renewable Energy, $1^{\text {st }}$ Edition, Elsevier Science, USA (2015).

[2] A. Paone, M. Joly, R. Sanjines, A. Romanyuk and J.-L. Scartezzini et al. Thermochromic films of VO2: W for smart solar energy applications. SPIE Optics \& Photonics Conference, San Diego, California, USA (2009).

[3] M. Joly, O. Bouvard, T. Gascou, Y. Antonetti and M. Python et al. Optical and structural analysis of sol-gel derived $\mathrm{Cu}-\mathrm{Co}-\mathrm{Mn}-\mathrm{Si}$ oxides for black selective solar nanocomposite multilayered coatings, Solar Energy Materials and Solar Cells, 143, 573-580 (2015).

[4] C-C. Chen, Visibly transparent polymer solar cells produced by solution processing, ACS Nano, 6 (8), pp 7185-7190 (2012).

[5] M. Joly, Y. Antonetti, M. Python, G. Lazo and M. Aymara et al. Selective solar absorber coatings on receiver tubes for CSP - energy-efficient production process by sol-gel dip-coating and subsequent induction heating. ISES Solar World Congress, Cancun, Mexico (2014).

[6] A. Schueler, D. Deepanshu, E. De Chambrier, C. Roecker and G. De Temmerman et al. Sol-gel deposition and optical characterization of multilayered $\mathrm{SiO}_{2} / \mathrm{Ti1}-\mathrm{xSixO}_{2}$ coatings on solar collector glasses, Solar Energy Materials \& Solar Cells, 90, 2894-2907 (2006).

[7] M. Joly, Y. Antonetti, M. Python, M. Gonzalez and T. Gascou et al. Energy-efficient sol-gel process for production of nanocomposite absorber coatings for tubular solar thermal collectors, CISBAT Conference, Lausanne, Switzerland (2013).

[8] S. U. S. Choi, J A Eastman, Enhancing thermal conductivity of fluids with nanoparticles, ASME International Mechanical Engineering Congress and Exposition, San Francisco, USA, (1995).

[9] O. Anwar Bég, Multi-physical computational modelling of nanofluid bioconvection flows in spacecraft bioreactors, A. Sohail, Z. Li (Eds.): Computational Approaches in Biomedical NanoEngineering, Wiley-CVH, China, Chapter 5, pp100-150 (2019). In press

[10] A.T. Brimmo et al., Stagnation-point flows in analytical chemistry and life sciences, $R S C A d v$., 7, 51206$51232(2017)$.

[11] N Bachok, A Ishak, I Pop, The boundary layers of an unsteady stagnation-point flow in a nanofluid, International Journal of Heat and Mass Transfer, 55, 6499-6505 (2012).

[12] Uddin, M.J., W.A. Khan, A.I.Md. Ismail, O. Anwar Bég, Computational study of threedimensional stagnation point nanofluid bio-convection flow on a moving surface with anisotropic slip and thermal jump effects, ASME J. Heat Transfer, 138(10), 104502-104509 (2016).

[13] R A Hamid, R Nazar, I Pop, Non-alignment stagnation-point flow of a nanofluid past a permeable stretching/shrinking sheet: Buongiorno's model, Scientific Reports, 5, 14640, 06.10.2015 (2015). 
[14] P. Duxson, G. C. Lukey and J. S. J. van Deventer, Evolution of gel structure during thermal processing of Na-geopolymer gels, Langmuir, 2006, 22 (21), pp 8750-8757.

[15] M. C. Scharber and N. S. Sariciftci, Efficiency of bulk-heterojunction organic solar cells, Progress in Polymer Science, 38, 1929-1940 (2013).

[16] R. Viskanta, Radiation heat transfer in materials processing and manufacturing, pp101-112, A. Bejan et al. (eds.), Energy and the Environment, 101-112, Kluwer Academic Publishers, New York (1999).

[17] S. Chandrasekhar, Radiative Transfer, Pergamon, New York, USA (1960).

[18] R. Siegel and J.R. Howell, Thermal Radiation Heat Transfer, MacGraw Hill, New York (1972).

[19] M Ferdows, MS Khan, O. Anwar Bég, MAK Azad, MM Alam, Numerical study of transient magnetohydrodynamic radiative free convection nanofluid flow from a stretching permeable surface, Proc. IMechE-Part E: J. Process Mechanical Engineering, 228 (3) 181-196 (2014).

[20] O. Anwar Bég, M. Ferdows, Tasveer A. Bég, T. Ahmed, M. Wahiduzzaman, Md. M. Alam, Radiative optically-dense magnetized transient reactive transport phenomena with cross diffusion and dissipation effects: numerical simulations, J. Taiwan Inst. Chemical Engineers, 66, 12-26 (2016).

[21] T. Thumma, O. Anwar Bég and Siva Reddy Sheri, Finite element computation of magnetohydrodynamic nanofluid convection from an oscillating inclined plate with radiative flux, heat source and variable temperature effects, Proc. IMechE- Part N-J. Nanoengineering Nanomaterials and Nanosystems, UK (2017). DOI: 10.1177/2397791417731452 (16 pages).

[22] L. S. Perše et al., Rheological and optical properties of solar absorbing paints with POSS-treated pigments, Materials Chemistry and Physics, 149, 368-377 (2015).

[23] L.S. Perše et al., The role of rheological properties and spraying parameters on the spectral selectivity of Thickness Insensitive Spectrally Selective (TISS) paint coatings, Solar Energy Materials and Solar Cells, 110, 115-125 (2013).

[24] S.Wijewardane D.Y.Goswami, A review on surface control of thermal radiation by paints and coatings for new energy applications, Renewable and Sustainable Energy Reviews, 16, 1863-1873 (2012).

[25] C. Atkinson et al., Coatings for concentrating solar systems - A review, Renewable and Sustainable Energy Reviews, 45, 113-122 (2015).

[26] Md. Jashim Uddin, N.H. Md. Yusoff, O. Anwar Beg and Ahamd Izani Ismail, Lie group analysis and numerical solutions for non-Newtonian nanofluid flow in a porous medium with internal heat generation, Physica Scripta 87 (2013) 025401 (14pp).

[27] M M Bhatti, M M Rashidi, Effects of thermo-diffusion and thermal radiation on Williamson nanofluid over a porous shrinking/stretching sheet, J. Molecular Liquids, 221, 567-573 (2016).

[28] T Hayat, N Gull, M Farooq, B Ahmad, Thermal radiation effects in MHD flow of Powell-Eyring nanofluid induced by a stretching cylinder, ASCE J. Aerospace Engineering, 29, 20-30 (2016).

[29] A.B. Huda, N.S. Akbar, O. Anwar Bég, and M.Y. Khan, Dynamics of variable-viscosity nanofluid flow with heat transfer in a felixble vertical tube with propagating waves, Results in Physics, 7, 413425 (2017). 
[30] P. Rana, R. Bhargava, O. Anwar Bég and A. Kadir, Finite element analysis of viscoelastic nanofluid flow with energy dissipation and internal heat source/sink effects, Int. J. Applied Computational Mathematics, 3 (2), 1421-1447 (2017)

[31] V. R. Prasad, S. A. Gaffar and O. Anwar Bég, Heat and mass transfer of a nanofluid from a horizontal cylinder to a micropolar fluid, AIAA J. Thermophysics Heat Transfer, 29, 1, 127-139 (2015).

[32] O. V. Stoyanov et al.(Editors), Nanopolymers and Modern Materials: Preparation, Properties and Applications, CRC Press, Florida, USA (2013).

[33]_D Pal, G Mandal, Influence of Lorentz force and thermal radiation on heat transfer of nanofluids over a stretching sheet with velocity thermal slip, International Journal of Applied and Computational Mathematics, 2, 1-20 (2016).

[34] N. A. Latiff, M. J. Uddin, O. Anwar Bég and A.I.M. Ismail, Unsteady forced bioconvection slip flow of a micropolar nanofluid from a stretching/ shrinking sheet, Proc. IMECHE- Part N: J. Nanoengineering and Nanosystems, 230 (4) pp. 177-187 (2016).

[35] M.J. Uddin, W.A. Khan, and O. Anwar Bég, Bioconvection nanofluid slip flow past a wavy surface with applications in nano-biofuel cells Chin. J. Physics, 55, 2048-2063 (2017)

[36] M Turkyilmazoglu, Exact analytical solutions for heat and mass transfer of MHD slip flow in nanofluids, Chemical Engineering Science, 84, 182-187 (2012).

[37] N. Nagendra, A. Subba Rao, CH. Amanulla, M. Surya Narayana Reddy, O. Anwar Bég, Hydromagnetic non-Newtonian nanofluid transport phenomena past an isothermal vertical cone with partial slip: aerospace nanomaterial enrobing simulation; Heat Transfer-Asian Research (2017). DOI: 10.1002/htj.21299 (28 pages)

[38] W Ibrahim, B Shankar, MHD boundary layer flow and heat transfer of a nanofluid past a permeable stretching sheet with velocity, thermal and solutal slip boundary conditions, Computers and Fluids, 75, $1-10$ (2013).

[39] A K Abdul Hakeem, N Vishnu, G B Ganga, Magnetic field effect on second order slip flow of nanofluid over a stretching/shrinking sheet with thermal radiation effect, J. Magnetism and Magnetic Materials, 381, 243-257 (2015).

[40] R Dhana, P Rana, L Kumar, MHD mixed convection nanofluid flow and heat transfer over an inclined cylinder due to velocity and thermal slip effects: Buongiorno's model, Powder Technology, 288, 140-150 (2016).

[41] B Prabhakar, S Bandari, C K Kumar, Effects of inclined magnetic field and chemical reaction on flow of a Casson nanofluid with second order velocity slip and thermal slip over an exponentially stretching sheet, International Journal of Applied and Computational Mathematics, 3 (4) 2967-2985 (2017).

[42] J.L. Throne, Radiant Heat Transfer in Thermoforming, SPE Annual Technical Conference ANTEC'95, Brookfield, USA (1995).

[43] R.D. Cess, The interaction of thermal radiation with free convection heat transfer, Int. J. Heat Mass Transfer, 9, 1269-77 (1966). 
[44] O. Anwar Bég, N. Ali, A. Zaman, Eemaan T. A. Bég and Ayesha Sohail, Computational modelling of heat transfer in annular porous medium solar energy absorber with a P1-radiative differential approximation, J. Taiwan Inst. Chemical Eng., 66, 258-268 (2016).

[45] O. Anwar Bég, S. Kuharat, R. Mehmood and M. Babie, Anisotropic radiative heat transfer in nanodoped solar gel collectors: CFD study with gold, copper, titanium and silver oxide nano-particles, Applied Energy (2018). In preparation.

[46] M. Modest, Radiative Heat Transfer, McGraw-Hill, New York, USA (1993).

[47] W A Khan, O D Makinde, Z H Khan, Non-aligned MHD stagnation point flow of variable viscosity nanofluids past a stretching sheet with radiative heat, International Journal of Heat and Mass Transfer, 96, 525-534 (2016).

[48] Uddin MJ Alginahi Y Bég OA and Kabir MN. Numerical solutions for gyrotactic bioconvection in nanofluid-saturated porous media with Stefan blowing and multiple slip effects. Comp. Math Appl. $72,2562-2581$ (2016).

[49] M. A. Hamad, I Pop, A I M Ismail, Magnetic field effects on free convection flow of a nanofluid past a vertical semi-infinite flat plate, Nonlinear Analysis: Real World Applications, 12, 1338-1346 (2011).

[50] M.J. Uddin, O. Anwar Bég and N.S. Amin, Hydromagnetic transport phenomena from a stretching or shrinking nonlinear nanomaterial sheet with Navier slip and convective heating: a model for bionano-materials processing, J. Magnetism Magnetic Materials, 368, 252-261(2014).

[51] B. Bradie, A Friendly Introduction to Numerical Analysis, Pearson Prentice Hall, Upper Saddle River, New Jersey:, USA (2006).

[52] H.B. Keller, Numerical Solution of Two-Point Boundary Value Problems, SIAM Press Philadelphia, USA (1976).

[53] J.H. He, Variational iteration method-a kind of non-linear analytical technique: some examples, Int. J. Non-Linear Mechanics, 34, 699-708 (1999).

[54] S. A. El-Wakil and E. M. Abulwafa, Variational-Iterative Method for conductive-radiative heat transfer in spherical inhomogeneous medium, AIAA J. Thermophysics and Heat Transfer, 14, 612-615 (2000).

[55] O. Anwar Bég, Spectral, VIM, ADM and HAM approaches in burn injury bioheat transfer simulation, Technical Report-Gort Engovation-BIO-T-12-2013,75 pages, Trondheim, Norway, February (2013)

[56] O Anwar Bég, S.S. Motsa, M.N. Islam and M. Lockwood (2014a). Pseudo-spectral and variational iteration simulation of exothermically-reacting Rivlin-Ericksen viscoelastic flow and heat transfer in a rocket propulsion duct, Computational Thermal Sciences, 6, 2, 91-102.

[57] A. F. Elsayed and O. Anwar Bég. New computational approaches for biophysical heat transfer in tissue under ultrasonic waves: Variational iteration and Chebyshev spectral simulations, J. Mechanics Medicine Biology, 14, 3, 1450043.1-1450043.17 (2014).

[58] H. Chen et al., Rheological behaviour of nanofluids, New J. Phys. 9 367-380 (2007). 
[59] Lewis Wedgewood, Ketan Joshi Stagnation flow studies of polymer solutions in 2D system, Applied Rheology, 9, 174-182 (2003).

[60] C.Y. Lee and T.A. Tallmadge, The stagnation-point in free coating, AIChemEng J., 19, 865-866 (1973).

[61] Paullet, J., Weidman, P., Analysis of stagnation point flow toward a stretching sheet, Int. J. Nonlinear Mech., 42, 1084-1091 (2007).

[62] Mahapatra, T. R. et al., Oblique stagnation-point flow and heat transfer towards a shrinking sheet with thermal radiation, Meccanica, 47, 1325-1335 (2012).

[63] Lok, Y. Y. et al., Oblique stagnation slip flow of a micropolar fluid, Meccanica, 45, 187-198 (2010).

[64] M. Du and G.H. Tang, Optical property of nanofluids with particle agglomeration, Solar Energy, $122,864-872(2015)$.

[65] Z. Said et al., Radiative properties of nanofluids, Int. Comm. Heat Mass Tran., 46, 74-84 (2013).

[66] A. Paone, J.-L. Scartezzini and A. Schueler (Dirs.). Switchable Selective Absorber Coatings for Overheating Protection of Solar Thermal Collectors. EPFL, Lausanne, Switzerland (2013).

[67] O. Anwar Bég, M. Faisal Md Basir, M.J. Uddin, and A. I. Md. Ismail, Numerical study of slip effects on asymmetric bioconvective nanofluid flow in a porous microchannel with an expanding/contracting upper wall using Buongiorno's model, J. Mechanics in Medicine and Biology, 17 (5) 1750059 (28 pages) (2017).

[68] J. Buongiorno, Convective transport in nanofluids, ASME J. Heat Transfer, 128, 240-250 (2006).

[69] M.J. Uddin, O. Anwar Bég and A.I. Ismail, Radiative-convective nanofluid flow past a stretching/shrinking sheet with slip effects, AIAA J. Thermophysics Heat Transfer, 29, 3, 513-523 (2015).

[70] Nadeem, S, Mehmood, R, Akbar, NS. Oblique stagnation point flow of a Casson-nano fluid towards a stretching surface with heat transfer. J Comput. Theor. Nanoscience, 11, 1422-1432 (2014).

[71] S. Kuharat, Simulation of natural convection in enclosure-based spacecraft solar systems, $M S c$ Dissertation, Aerospace Engineering, Department of Aeronautical and Mechanical Engineering, University of Salford, Manchester, UK, 99pp, September (2017).

[72] L. Shi, Y. He, Y. Huang, B. Jiang, Recyclable $\mathrm{Fe}_{3} \mathrm{O}_{4} @ \mathrm{CNT}$ nanoparticles for high-efficiency solar vapor generation, Energy Conversion and Management 149, 401-408 (2017). 